Marquette University

e-Publications@Marquette

Social and Cultural Sciences Faculty Research and

Publications

Social and Cultural Sciences, Department of

6-1-2015

Single-Father Families: A Review of the Literature

Roberta L. Coles

Marquette University, roberta.coles@marquette.edu

Accepted version. Journal of Family Theory \& Review, Vol. 7, No. 2 (June 2015): 144-166. DOI. (C) 2015 John Wiley \& Sons, Inc. Used with permission. 


\title{
Single-Father Families: A Review of the Literature
}

\author{
Roberta L. Coles \\ Department of Sociology, Marquette University \\ Milwaukee, WI
}

\begin{abstract}
The number of children residing in single-father families in the United States quadrupled as a proportion of children's living arrangements during the past few decades of the 20th century. Research on single fathers also increased and changed in nature. This article is a review of the research on single fathers and their families from the 1970s until recently, focusing on modifications in methodology and theoretical underpinnings. In general, research on single-father families evolved from qualitative studies focused on the well-being of single fathers to quantitative studies focusing on child outcomes and within-group variation among single fathers. Research also moved from descriptive studies to those testing gender and microstructural theories. This article also summarizes the main findings on single fathers and concludes with directions for future research.
\end{abstract}

The announcements have been coming for several decades now-single fathers are increasing in the United States, particularly since 1960. However, there are many ways to slice and dice the numbers. One thing that needs to be kept in mind when reading data on the rise in US single-father households is whether researchers are measuring the number or percentage of single-father households or the number or percentage of children living in such households. A Pew 
Research Center Report (Livingston, 2013) recently announced that "the number of single father households has increased about nine-fold since 1960, from less than 300,000 to more than 2.6 million in 2011. In comparison, the number of single-mother households increased more than fourfold during that time period" (n.p.). It is not surprising that the number has increased; the population has increased.

Increases in number, therefore, are not equivalent to an increase in rate. Even when the percentage of single-father families increases as a portion of all families, as it has done, part of that increase is because the pool of family households has decreased as a portion of all households. That is, in 1960 family households represented about $85 \%$ of all US households; today they represent about $66 \%$, because nonfamily households (single persons and cohabitors without children) have increased (US Census, 2013b). Therefore, the rate of one-parent father households has increased about fourfold since 1960 (US Census, 2013c). However, the percentage of children being raised in those households has increased at a slower rate.

Another way to measure single fatherhood is according to the percentage of children living with their father only, which is longerterm data from the US Census Bureau. That data indicate that the proportion of children living with fathers increased nearly threefold since the 1960 s, from $1.3 \%$ in 1960 to $3.7 \%$ in 2009 , but the period 1950- 1980 was a historic low in father-only parenting. In 1880, 2.6\% of children under 18 were living in father-only families, and in 1920, $2.9 \%$ were (US Census, 2011), making the long-term increase less than twofold.

Another measurement issue that may contribute to an inflated increase in single fathers is that the census definition of father-only families includes fathers who cohabit with romantic partners. As Table 1 shows, about $22 \%$ of children living in father-only households are living with cohabiting fathers. (This does not include single fathers who are cohabiting with the biological mothers; those households are included under two-parent households.) Including cohabiting single fathers likely increases the number and percentage of men included as father-only families. 
Table 1. Characteristics of Father-Only and Mother-Only Households, $2013^{*}$

Characteristics of household
Children (under 18) in Children (under 18) in father-only households mother-only households (\%) $\underline{a}$
(\%) $\underline{\mathbf{b}}$

- Note. Total number of children (under 18) in US =73,910,000. Table data calculated from Table C3. Living Arrangements of Children Under 18 Years (excluding group quarters) and Marital Status of Parents, by Age, Sex, Race, and Hispanic Origin, and Selected Characteristics of the Child for All Children: 2013. US Census Bureau, Current Population Survey, 2013 Annual Social and Economic Supplement.

- aTotal number of children in father-only households $=2,998,000$, or $4.1 \%$.

- bTotal number of children in mother-only households $=17,532,000$, or $23.7 \%$

\begin{tabular}{|c|c|c|}
\hline \multicolumn{3}{|l|}{ Marital status of parent } \\
\hline $\begin{array}{l}\text { Married, spouse } \\
\text { absent }\end{array}$ & 4.5 & 5.1 \\
\hline Widowed & 4.4 & 2.9 \\
\hline Divorced & 43.8 & 29.9 \\
\hline Separated & 16.7 & 14.1 \\
\hline Never married & 30.6 & 47.9 \\
\hline \multicolumn{3}{|l|}{ Household configuration } \\
\hline $\begin{array}{l}\text { No other adults in } \\
\text { household }\end{array}$ & 51.6 & 56 \\
\hline $\begin{array}{l}\text { Cohabiting, not with } \\
\text { mother }\end{array}$ & 22.4 & 10.5 \\
\hline $\begin{array}{l}\text { Other relatives } \\
\text { present }\end{array}$ & 21.3 & 29.7 \\
\hline Male children & 53.2 & 50.4 \\
\hline Children 5 or under & 23.2 & 30.6 \\
\hline 1 child & 35.4 & 24.3 \\
\hline$\geq 2$ siblings & 26.3 & 39.1 \\
\hline \multicolumn{3}{|l|}{ Parental characteristics } \\
\hline $\begin{array}{l}\text { Some college or more } \\
\text { education }\end{array}$ & 50.6 & 52.4 \\
\hline $\begin{array}{l}\text { Income of } \$ 30 \mathrm{~K} \text { or } \\
\text { more }\end{array}$ & 66.6 & 43.0 \\
\hline $\begin{array}{l}\text { At or below poverty } \\
\text { threshold }\end{array}$ & 21.2 & 44.6 \\
\hline
\end{tabular}

Journal of Family Theory \& Review, Vol 7, No. 2 (June 2015): pg. 144-166. DOI. This article is (C John Wiley \& Sons, Inc. and permission has been granted for this version to appear in e-Publications@Marquette. John Wiley \& Sons, Inc does not grant permission for this article to be further copied/distributed or hosted elsewhere without the express permission from John Wiley \& Sons, Inc. 
No matter what the exact increase is, several legal and cultural factors have played a role in producing it. The concept of fatherhood has changed so that more "parenting," not only the provision of income, is expected of fathers (Taylor, Parker, Morin, Cohn, \& Wang, 2013). Laws governing divorce and custody have changed in ways designed to treat spouses and parents more equally, and they have thus facilitated more father custody (although several studies indicate that most single fathers obtain custody through out-of-court agreements between parents) (Coles, 2009; Hamer \& Marchioro, 2002; Pearson, Munson, \& Thoennes, 1982). Single men are now allowed to adopt children (usually older children), but these adoptions, whether by gay or straight men, account for a very small percentage of single fathers. Adoption statistics are hard to come by because states are not required to keep record of them. However, it is estimated that in recent years, about 3\% of adoptions through foster care have been by single men, often gay, and adoptions by single men through foster care are usually of older, harder-to-place children (Koch, 2007). In 2007, the Williams Institute estimated that about 65,000 adopted children were being raised in LGBT homes, but many of these are households headed by lesbian women (Gates, Badgett, Macomber, \& Chambers, 2007).

The most significant contributing factors have been demographic in nature. Until the 1980s, divorce and, secondarily, widowhood largely accounted for single fatherhood. Divorce has since declined from its 1980 peak, but it has leveled off at a relatively high rate and thus remains a primary contributing factor. In 1980 the proportion of widowed single fathers was surpassed by never-married single fathers. Nonmarital births accounted for about 41\% of all births in 2011 (Child Trends Databank, 2013). This trend intersects with the growing popularity of cohabitation, as the majority of nonmarital births occur with cohabitation (Child Trends Databank, 2013). Thus, a much greater percentage of single fathers attain their role following nonmarital births, whether or not they cohabit. As Table $\underline{1}$ shows, according to 2013 census data, children in never-married fatherhouseholds are now the second-largest group of children (30.6\%) in father-only households, and widowed (4.4\%) households are the smallest group. 
Research on single fathers has mirrored the increase in single fathering, evolving over the years in quantity and quality. This article reviews the research on single fathers and their families from the 1970s, focusing on changes in methodology and theoretical underpinnings over the years. The article also summarizes the main findings on what we know about single fathers, and it concludes with directions for future theorizing, research, and practice.

It is important to note here that the terms designating single fathers vary, so who qualifies as a single father may vary from one study to the next. The Census Bureau uses the terms father-only households or male-headed households with own children. As mentioned earlier, these labels include men who cohabit with someone other than the child's mother $(22.4 \%)$, cohabit with other relatives $(21.3 \%)$, or are currently married but are separated $(16.7 \%)$, or the spouse is absent for some reason (4.5\%) (see Table 1 ). In the Pew report mentioned earlier (Livingston, 2013), single fathers included unmarried men who reported that their children had been living with them for at least two months, and $41 \%$ of the fathers were cohabiting. It is unclear in the report what percentage, if any, of cohabiting partners were the biological mother.

Some researchers study "custodial fathers," which often means divorced fathers who have custody of children, and some of those fathers may be remarried. For instance, in Grall's (2011) study of custodial parents' receipt of child support, which relied on census data, about $22 \%$ of custodial fathers were married (compared to $18 \%$ of custodial mothers). However, most of the time, custodial just means the father has custody, legal or otherwise, of one or more of his children. Occasionally, single father is used to refer to nonresident fathers, although I excluded such studies from this review. Throughout this review I have used single fathers generally to refer to men, cohabiting or not, who are coresiding with their children, separately from the child's mother. But when discussing specific research, I have tried to employ the terms used by the researchers.

Some claim that fathers who cohabit or live with extended family are not truly single fathers, as if they were cheating some idealized type of single fatherhood, but they are legally single, and single mothers are measured in the same way (although usually a 
smaller percentage of them are cohabiting, and a higher percentage lives with extended family; see Table $\underline{1}$ ). It remains to each specific study to determine the extent to which the fathers are coparenting with their new spouses or cohabiting partners or other adults and the extent to which coparenting is beneficial to the fathers and the children (some research seems to indicate that it is not that beneficial). In addition, keep in mind that even fathers residing alone with their children are often coparenting with nonresident mothers or other helpful adults. Let's also keep in mind that single mothers who are also coparenting or getting help from other adults don't usually have their "single parent" status questioned. Finally, it is often integral to the research question to include single and custodial fathers who are married or cohabiting. Grall's (2011) study needed to compare the effect of marriage on receipt of child support; other studies have aimed to detect the effects of the presence of other adults on single fathers and their children. Most research to date, particularly quantitative data sets, for which data are originally collected with some other purpose in mind, has not found a way to fully capture all these possible scenarios and their repercussions simultaneously. Hence, it is up to researchers to be aware of and weed out various father categories according to the needs of the research question and to clarify differences among fathers' residences in their conclusions. It is readers' responsibility to discern how the research defines and counts "single father."

The articles included in this review were obtained by searching for the keywords single father in social science databases, such as JSTOR and Academic Elite. A few secondary articles were added if they were repeatedly cited in the first-level articles. A few of those secondary articles actually stemmed from research focusing on "family structure," but they included a sample of single fathers. (For a chronological listing of the studies included in this review, along with the basic theory, method, and data used, see Table 2.)

Table 2. Characteristics of Research Studies

\begin{tabular}{|c|l|l||l|l||l|}
\hline Year & Author(s) & Focus & Theory & Method/ Data & $\begin{array}{c}\text { Single- } \\
\text { Father } \\
\text { Type(s) }\end{array}$ \\
\hline
\end{tabular}

1. Note. Divorced includes separated in most studies. 
NOT THE PUBLISHED VERSION; this is the author's final, peer-reviewed manuscript. The published version may be accessed by following the link in the citation at the bottom of the page.

\begin{tabular}{|c|c|c|c|c|c|c|}
\hline Year & Author(s) & Focus & Theory & Method/ Data & Sample & $\begin{array}{c}\text { Single- } \\
\text { Father } \\
\text { Type(s) }\end{array}$ \\
\hline 1976 & $\begin{array}{l}\text { Gasser \& } \\
\text { Taylor }\end{array}$ & $\begin{array}{l}\text { Father } \\
\text { adjustment }\end{array}$ & None applied (N/A) & $\begin{array}{l}\text { Qualitative } \\
\text { (Qual) }\end{array}$ & $\begin{array}{l}40 \text { single } \\
\text { fathers (SF) }\end{array}$ & \begin{tabular}{|l} 
Divorced or \\
widowed. Race \\
unspecified.
\end{tabular} \\
\hline $1976 a$ & Mendes & $\begin{array}{l}\text { Father } \\
\text { adjustment }\end{array}$ & $N / A$ & Qual & $32 \mathrm{SF}$ & \begin{tabular}{|l} 
Divorced, \\
widowed. 47\% \\
Black, 44\% \\
White. \\
Outcomes not \\
specified by \\
race.
\end{tabular} \\
\hline $1976 b$ & Mendes & $\begin{array}{l}\text { Father } \\
\text { adjustment }\end{array}$ & $N / A$ & Qual & $32 \mathrm{SF}$ & \begin{tabular}{|l} 
Divorced, \\
widowed. 47\% \\
Black, 44\% \\
White. \\
Outcomes not \\
specified by \\
race.
\end{tabular} \\
\hline 1976 & \begin{tabular}{|l} 
Orthner, \\
Brown, \& \\
Ferguson
\end{tabular} & $\begin{array}{l}\text { Father } \\
\text { adjustment }\end{array}$ & $\mathrm{N} / \mathrm{A}$ & Qual & $\begin{array}{l}20 \text { SF } \\
\text { North } \\
\text { Carolina }\end{array}$ & \begin{tabular}{|l} 
Divorced, \\
widowed, \\
never married. \\
Race \\
unspecified.
\end{tabular} \\
\hline 1979 & \begin{tabular}{|l} 
Orthner \& \\
Lewis
\end{tabular} & $\begin{array}{l}\text { Father } \\
\text { Competence }\end{array}$ & $\mathrm{N} / \mathrm{A}$ & Meta-analysis & \begin{tabular}{|l|}
5 studies \\
$1976-1979$
\end{tabular} & $\begin{array}{l}\text { SF, but } \\
\text { unspecified }\end{array}$ \\
\hline 1979 & \begin{tabular}{|l|} 
Santrock \& \\
Warshak
\end{tabular} & \begin{tabular}{|l|} 
Children's \\
social \\
development
\end{tabular} & $\begin{array}{l}\text { Same-sex parent- } \\
\text { child }\end{array}$ & \begin{tabular}{|l} 
Qual \& \\
quantitative \\
(Quant) \\
Videotapes \\
Texas
\end{tabular} & \begin{tabular}{|l}
60 families: \\
$20 \mathrm{SF}$ \\
20 single \\
mothers \\
$(\mathrm{SM})$ \\
20 2-parent \\
$(2-p)$
\end{tabular} & $\begin{array}{l}\text { Divorced } \\
\text { All White }\end{array}$ \\
\hline 1981 & \begin{tabular}{|l} 
DeFrain \& \\
Eirick
\end{tabular} & $\begin{array}{l}\text { Parental } \\
\text { adjustment }\end{array}$ & $\mathrm{N} / \mathrm{A}$ & $\begin{array}{l}\text { Qual } \\
\text { Nebraska }\end{array}$ & $\begin{array}{l}38 \mathrm{SF} \\
38 \mathrm{SM}\end{array}$ & $\begin{array}{l}\text { Divorced } \\
\text { All White } \\
\end{array}$ \\
\hline 1981 & \begin{tabular}{|l} 
Rosenthal \\
\& Keshet
\end{tabular} & $\begin{array}{l}\text { Father } \\
\text { adjustment }\end{array}$ & $\mathrm{N} / \mathrm{A}$ & Qual & $49 \mathrm{SF}$ & \begin{tabular}{|l} 
Divorced. \\
Caring for \\
children 2+ \\
days a week. \\
Race \\
unspecified.
\end{tabular} \\
\hline 1981 & \begin{tabular}{|l} 
Smith \& \\
Smith
\end{tabular} & $\begin{array}{l}\text { Father } \\
\text { experience }\end{array}$ & $N / A$ & Qual & $27 \mathrm{SF}$ & \begin{tabular}{|l} 
Divorced \& \\
widowed. All \\
White.
\end{tabular} \\
\hline
\end{tabular}

Journal of Family Theory \& Review, Vol 7, No. 2 (June 2015): pg. 144-166. DOI. This article is @ John Wiley \& Sons, Inc. and permission has been granted for this version to appear in e-Publications@Marquette. John Wiley \& Sons, Inc does not grant permission for this article to be further copied/distributed or hosted elsewhere without the express permission from John Wiley \& Sons, Inc. 
NOT THE PUBLISHED VERSION; this is the author's final, peer-reviewed manuscript. The published version may be accessed by following the link in the citation at the bottom of the page.

\begin{tabular}{|c|c|c|c|c|c|c|}
\hline Year & Author(s) & Focus & Theory & Method/ Data & Sample & $\begin{array}{l}\text { Single- } \\
\text { Father } \\
\text { Type(s) }\end{array}$ \\
\hline 1982 & Ambert & $\begin{array}{l}\text { Child } \\
\text { behavior } \\
\text { toward } \\
\text { parent }\end{array}$ & $N / A$ & Qual & $\begin{array}{l}20 \mathrm{SM} \\
7 \mathrm{SF} \\
\text { Toronto }\end{array}$ & 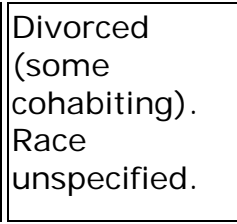 \\
\hline 1982 & \begin{tabular}{|l} 
Chang \& \\
Deinard
\end{tabular} & $\begin{array}{l}\text { Father } \\
\text { adjustment }\end{array}$ & $\mathrm{N} / \mathrm{A}$ & Survey & $80 \mathrm{SF}$ & \begin{tabular}{|l} 
Divorced, 90\% \\
White
\end{tabular} \\
\hline 1983 & $\begin{array}{l}\text { Warshak \& } \\
\text { Santrock }\end{array}$ & $\begin{array}{l}\text { Children's } \\
\text { outcomes }\end{array}$ & $\begin{array}{l}\text { Same-sex parent- } \\
\text { child }\end{array}$ & $\begin{array}{l}\text { Qual \& Quant } \\
\text { videotapes } \\
\text { Texas }\end{array}$ & $\begin{array}{l}60 \text { families: } \\
20 \mathrm{SF} \\
20 \mathrm{SM} \\
20 \text { 2-p }\end{array}$ & \begin{tabular}{|l} 
Divorced. All \\
White
\end{tabular} \\
\hline 1984 & $\begin{array}{l}\text { Gladding \& } \\
\text { Huber }\end{array}$ & $\begin{array}{l}\text { Father } \\
\text { profile }\end{array}$ & $\mathrm{N} / \mathrm{A}$ & Review & \begin{tabular}{|l}
$\begin{array}{l}\text { 1970s- } \\
1980 s \\
\text { research }\end{array}$ \\
\end{tabular} & Varied \\
\hline 1986 & $\begin{array}{l}\text { Norton \& } \\
\text { Glick }\end{array}$ & Profile & $\mathrm{N} / \mathrm{A}$ & \begin{tabular}{|l} 
University of \\
Michigan's \\
Panel Study of \\
Income \\
Dynamics
\end{tabular} & $\begin{array}{l}24,3392-p \\
5,907 \mathrm{SM} \\
800 \mathrm{SF}\end{array}$ & $\begin{array}{l}\text { Demographic } \\
\text { profile by } \\
\text { race. }\end{array}$ \\
\hline 1986 & Risman & \begin{tabular}{|l} 
Father \\
competency \\
\& \\
satisfaction. \\
Parent-child \\
closeness \\
\end{tabular} & $\begin{array}{l}\text { Gender v. } \\
\text { microstructural }\end{array}$ & Quant & $\begin{array}{l}121 \text { SF } \\
\text { NE United } \\
\text { States }\end{array}$ & \begin{tabular}{|l} 
Prior marital \\
status \\
unspecified. \\
$90 \%$ White.
\end{tabular} \\
\hline 1987 & Risman & \begin{tabular}{|l} 
Parental \\
behaviors
\end{tabular} & $\begin{array}{l}\text { Gender vs. } \\
\text { microstructural }\end{array}$ & Quant & $\begin{array}{l}55 \text { SF } \\
73 \text { SM } \\
155 \\
\text { married } \\
\text { couples } \\
\text { NE United } \\
\text { States }\end{array}$ & \begin{tabular}{|l} 
SF = widowed \\
or deserted. \\
Mostly White. \\
Outcomes not \\
specified by \\
race.
\end{tabular} \\
\hline 1988 & $\begin{array}{l}\text { McLanahan } \\
\text { \& Bumpass }\end{array}$ & \begin{tabular}{|l} 
Family \\
structure \\
effect on \\
adult \\
outcomes
\end{tabular} & $\begin{array}{l}\text { Intergenerational } \\
\text { instability due to } \\
\text { economic } \\
\text { deprivation, role, } \\
\text { stress, or selection }\end{array}$ & $\begin{array}{l}\text { Quant: } 1982 \\
\text { NSFG }\end{array}$ & $\begin{array}{l}7,969 \text { adult } \\
\text { women }\end{array}$ & \begin{tabular}{|l} 
Whether from \\
2 -parent or \\
single parent, \\
due to death, \\
divorce, \& \\
never married \\
$60 \%$ White, \\
$40 \%$ Black. \\
\end{tabular} \\
\hline
\end{tabular}

Journal of Family Theory \& Review, Vol 7, No. 2 (June 2015): pg. 144-166. DOI. This article is @ John Wiley \& Sons, Inc. and permission has been granted for this version to appear in e-Publications@Marquette. John Wiley \& Sons, Inc does not grant permission for this article to be further copied/distributed or hosted elsewhere without the express permission from John Wiley \& Sons, Inc. 


\begin{tabular}{|c|c|c|c|c|c|c|}
\hline Year & Author(s) & Focus & Theory & Method/ Data & Sample & $\begin{array}{c}\text { Single- } \\
\text { Father } \\
\text { Type(s) }\end{array}$ \\
\hline & & & & & & \begin{tabular}{||l} 
Outcomes \\
similar by \\
race, except \\
no family \\
structure \\
effect for \\
Blacks on \\
early \\
marriage. \\
Otherwise, \\
effect size \\
larger for \\
Whites.
\end{tabular} \\
\hline 1988 & $\begin{array}{l}\text { Risman \& } \\
\text { Park }\end{array}$ & \begin{tabular}{|l} 
Parent-child \\
relations
\end{tabular} & \begin{tabular}{|l|} 
Gender/individualist \\
vs. microstructural
\end{tabular} & Quant & $\begin{array}{l}148 \mathrm{SF} \\
73 \mathrm{SM}\end{array}$ & \begin{tabular}{|l}
$\begin{array}{l}\text { SF = widowed, } \\
\text { divorced, } \\
\text { deserted. }\end{array}$ \\
Race \\
unspecified.
\end{tabular} \\
\hline 1992 & \begin{tabular}{|l|} 
Thomson, \\
McLanahan, \\
\& Curtin
\end{tabular} & \begin{tabular}{|l} 
Parental \\
socialization
\end{tabular} & $\begin{array}{l}\text { Gender vs. } \\
\text { structural }\end{array}$ & $\begin{array}{l}\text { Quant: } 1987- \\
\text { 88 NSFH }\end{array}$ & $\begin{array}{l}3,738 \\
\text { parents of } \\
\text { children } \\
\text { ages 5- } 18\end{array}$ & $\begin{array}{l}\text { 2-p, SF, SM, } \\
\text { w/stepparents. } \\
\text { Divorced, } \\
\text { widowed, } \\
\text { never married. } \\
\text { Race } \\
\text { unspecified. }\end{array}$ \\
\hline 1993 & \begin{tabular}{|l} 
Downey \& \\
Powell
\end{tabular} & $\begin{array}{l}\text { Child } \\
\text { outcomes }\end{array}$ & Same-sex & $\begin{array}{l}\text { Quant: 1987- } \\
\text { 88 NELS }\end{array}$ & \begin{tabular}{|l}
, 892 8th \\
graders in \\
M-only \& F- \\
only \\
households
\end{tabular} & \begin{tabular}{|l} 
Divorced, \\
widowed, \\
never married. \\
Race \\
unspecified. \\
\end{tabular} \\
\hline 1993 & \begin{tabular}{|l} 
Meyer \& \\
Garasky
\end{tabular} & \begin{tabular}{|l} 
Child \\
support \\
receipt
\end{tabular} & $N / A$ & \begin{tabular}{|l} 
Quant: 1987 \\
CPS, 1986 \\
SIPP
\end{tabular} & $\begin{array}{l}814 \mathrm{SF} \\
385 \mathrm{CF} \\
16,402 \mathrm{MF} \\
4,937 \mathrm{SM} \\
2,114 \mathrm{CM}\end{array}$ & \begin{tabular}{|l} 
Excluded \\
those with \\
other adults \\
and widowed. \\
$>80 \%$ White. \\
Outcomes not \\
specified by \\
race.
\end{tabular} \\
\hline 1994 & Downey & \begin{tabular}{|l|} 
lhildren's \\
school \\
performance
\end{tabular} & $\begin{array}{l}\text { Microstructure vs. } \\
\text { gender }\end{array}$ & $\begin{array}{l}\text { Quant: } 1988 \\
\text { NELS }\end{array}$ & \begin{tabular}{|l} 
th \\
graders: \\
409 in $S F$ \\
3,483 in \\
SM, \& \\
14,269 biol. \\
$2-p$
\end{tabular} & $\begin{array}{l}\text { Cohabiting \& } \\
\text { step excluded. } \\
\text { Outcomes not } \\
\text { specified by } \\
\text { race. }\end{array}$ \\
\hline
\end{tabular}

Journal of Family Theory \& Review, Vol 7, No. 2 (June 2015): pg. 144-166. DOI. This article is @ John Wiley \& Sons, Inc. and permission has been granted for this version to appear in e-Publications@Marquette. John Wiley \& Sons, Inc does not grant permission for this article to be further copied/distributed or hosted elsewhere without the express permission from John Wiley \& Sons, Inc. 
NOT THE PUBLISHED VERSION; this is the author's final, peer-reviewed manuscript. The published version may be accessed by following the link in the citation at the bottom of the page.

\begin{tabular}{|c|c|c|c|c|c|c|}
\hline Year & Author(s) & Focus & Theory & Method/ Data & Sample & $\begin{array}{l}\text { Single- } \\
\text { Father } \\
\text { Type(s) }\end{array}$ \\
\hline 1995 & $\begin{array}{l}\text { Hall, } \\
\text { Walker, \& } \\
\text { Acock }\end{array}$ & \begin{tabular}{|l} 
Parental \\
involvement
\end{tabular} & $\begin{array}{l}\text { Gender, } \\
\text { microstructural }\end{array}$ & $\begin{array}{l}\text { Quant: 1987- } \\
\text { 1988 NSFH }\end{array}$ & $\begin{array}{l}1,433 \mathrm{SM} \\
128 \mathrm{SF}\end{array}$ & $\begin{array}{l}\text { Divorced, } \\
\text { widowed, } \\
\text { never married } \\
\text { SF = 80\% } \\
\text { White, } 12 \% \\
\text { Black. } \\
\text { Outcomes not } \\
\text { specified by } \\
\text { race }\end{array}$ \\
\hline 1996 & \begin{tabular}{|l} 
Clarke- \\
Stewart \& \\
Hayward
\end{tabular} & $\begin{array}{l}\text { Children's } \\
\text { psychological } \\
\text { well-being }\end{array}$ & Same-sex & $\begin{array}{l}\text { Quant: } \\
\text { Southern CA } \\
\text { sample }\end{array}$ & $\begin{array}{l}187 \\
\text { children }\end{array}$ & \begin{tabular}{|l} 
Divorced, \\
mother or \\
father \\
Custody. $80 \%$ \\
white
\end{tabular} \\
\hline 1996 & $\begin{array}{l}\text { Cooksey \& } \\
\text { Fondell }\end{array}$ & $\begin{array}{l}\text { Time spent } \\
\text { w/children \& } \\
\text { academic } \\
\text { achievement }\end{array}$ & $\begin{array}{l}\text { Evolutionary vs. } \\
\text { microstructural \& } \\
\text { gender }\end{array}$ & $\begin{array}{l}\text { Quant: 1987- } \\
1988 \text { NSFH }\end{array}$ & $\begin{array}{l}1,250 \\
\text { fathers } \\
\text { w/children } \\
\text { ages 5-18 }\end{array}$ & \begin{tabular}{|l} 
2-parent, \\
biological, \\
step. SF \& SF \\
w/stepmom, \\
stepfather, \& \\
SM. \\
\end{tabular} \\
\hline 1996 & \begin{tabular}{|l} 
Eggebeen, \\
Snyder, \& \\
Manning
\end{tabular} & $\begin{array}{l}\text { Demographic } \\
\text { profile over } \\
\text { time }\end{array}$ & $\mathrm{N} / \mathrm{A}$ & \begin{tabular}{|l} 
Quant: 1960, \\
1970, 1980, \\
1990 PUMS
\end{tabular} & $\begin{array}{l}\text { 1960: 703; } \\
\text { 1970: } \\
1,250 ; \\
1980: \\
1,291 ; \\
1990: \\
115,972\end{array}$ & $\begin{array}{l}9 \text { types of } \\
\text { single fathers: } \\
\text { lone, complex, } \\
\text { cohabiting, } \\
\text { divorced, } \\
\text { widowed, } \\
\text { never married }\end{array}$ \\
\hline 1996 & \begin{tabular}{|l} 
Greif \& \\
DeMaris
\end{tabular} & Profile & $N / A$ & \begin{tabular}{|l} 
Quant: 1987- \\
1988 Survey \\
of Parents w/o \\
Partners
\end{tabular} & $933 \mathrm{SF}$ & $\begin{array}{l}\text { Divorced. 96\% } \\
\text { White }\end{array}$ \\
\hline 1997 & \begin{tabular}{|l} 
Powell \& \\
Downey
\end{tabular} & \begin{tabular}{|l} 
Child well- \\
being
\end{tabular} & Same-sex & \begin{tabular}{|l} 
Quant: NELS, \\
high school \\
and beyond, \\
and GSS
\end{tabular} & \begin{tabular}{|l}
1,205 teens \\
in SF \& \\
6,089 in \\
SM. 3,724 \\
adult \\
children
\end{tabular} & $\begin{array}{l}\text { Father-only \& } \\
\text { mother-only, } \\
\text { no other adult }\end{array}$ \\
\hline 1998 & \begin{tabular}{|l} 
Downey, \\
Ainsworth- \\
Darnell, \& \\
Dufur \\
\end{tabular} & $\begin{array}{l}\text { Children's } \\
\text { well-being }\end{array}$ & $\begin{array}{l}\text { Gender vs. } \\
\text { structural }\end{array}$ & \begin{tabular}{|l|} 
Quant: 1990 \\
NELS \& 1972- \\
1994 GSS
\end{tabular} & $\begin{array}{l}3,039 \text { ages } \\
15-16\end{array}$ & $\begin{array}{l}456 \text { in SF, no } \\
\text { other adult. } \\
2,583 \text { in SM, } \\
\text { no other adult }\end{array}$ \\
\hline 1998 & $\begin{array}{l}\text { Guttman \& } \\
\text { Lazar }\end{array}$ & $\begin{array}{l}\text { Children's } \\
\text { social } \\
\text { adjustment }\end{array}$ & Same-sex & Quant & \begin{tabular}{|l}
31 boys \\
and 28 girls \\
in junior \\
high
\end{tabular} & \begin{tabular}{|l} 
Boys \& girls \\
from single \\
divorced \\
parents, and \\
2-p families
\end{tabular} \\
\hline 1998 & $\begin{array}{l}\text { Hilton \& } \\
\text { Devall }\end{array}$ & $\begin{array}{l}\text { Children's } \\
\text { behavior }\end{array}$ & Ecological theory & $\begin{array}{l}\text { Quant: data } \\
\text { from 2 } \\
\text { elementary }\end{array}$ & $30 \mathrm{SM}$ & \begin{tabular}{|l} 
Divorced, 90\% \\
white. \\
Outcomes
\end{tabular} \\
\hline
\end{tabular}

Journal of Family Theory \& Review, Vol 7, No. 2 (June 2015): pg. 144-166. DOI. This article is (C) John Wiley \& Sons, Inc. and permission has been granted for this version to appear in e-Publications@Marquette. John Wiley \& Sons, Inc does not grant permission for this article to be further copied/distributed or hosted elsewhere without the express permission from John Wiley \& Sons, Inc. 
NOT THE PUBLISHED VERSION; this is the author's final, peer-reviewed manuscript. The published version may be accessed by following the link in the citation at the bottom of the page.

\begin{tabular}{|c|c|c|c|c|c|c|}
\hline Year & Author(s) & Focus & Theory & Method/ Data & Sample & $\begin{array}{l}\text { Single- } \\
\text { Father } \\
\text { Type(s) }\end{array}$ \\
\hline & & & & $\begin{array}{l}\text { schools in } \\
\text { Southwest }\end{array}$ & $\begin{array}{l}30 \text { SF } \\
30 \text { 2-parent }\end{array}$ & $\begin{array}{l}\text { unspecified by } \\
\text { race. }\end{array}$ \\
\hline 1998 & $\begin{array}{l}\text { Hoffmann \& } \\
\text { Johnson }\end{array}$ & $\begin{array}{l}\text { Adolescent } \\
\text { drug use }\end{array}$ & $\begin{array}{l}\text { Economic } \\
\text { resources, mobility }\end{array}$ & $\begin{array}{l}\text { Quant: } \\
\text { NHSDA }\end{array}$ & $\begin{array}{l}22,237 \\
\text { adolescents }\end{array}$ & \begin{tabular}{|l} 
2-parent. \\
biological, M \& \\
stepfather, \\
father \& \\
stepmother, \\
mother-only, \\
father-only, \\
mother \& \\
other relative. \\
$69 \%$ White, \\
$15 \%$ Black, \\
$12 \%$ Hispanic. \\
Outcomes not \\
specified by \\
race.
\end{tabular} \\
\hline 1999 & Cookston & \begin{tabular}{|l} 
Parental \\
supervision \\
$\&$ adolescent \\
behavior
\end{tabular} & $\mathrm{N} / \mathrm{A}$ & Quant: NLSAH & $\begin{array}{l}684 \\
\text { adolescents }\end{array}$ & \begin{tabular}{|l} 
SM vs. SF v 2- \\
p. $64 \%$ White, \\
$27 \%$ Black, \\
$2 \%$ AlAN, \\
$2.5 \%$ Asian \\
American, but \\
outcomes \\
unspecified
\end{tabular} \\
\hline 1999 & \begin{tabular}{|l} 
Heath \& \\
Orthner
\end{tabular} & $\begin{array}{l}\text { Parental } \\
\text { coping }\end{array}$ & $\begin{array}{l}\text { Family adaptation } \\
\text { perspective }\end{array}$ & $\begin{array}{l}\text { Quant: } 1995 \\
\text { US Air Force } \\
\text { sample }\end{array}$ & $\begin{array}{l}346 \text { SF } \\
364 \text { SM }\end{array}$ & $\begin{array}{l}\text { Divorced, } \\
\text { never married } \\
\text { Race not } \\
\text { specified }\end{array}$ \\
\hline 1999 & Hill \& Hilton & $\mid \begin{array}{l}\text { Parental } \\
\text { well-being }\end{array}$ & Role theory & $\begin{array}{l}\text { Quant: 1987- } \\
\text { 1988 NSFH }\end{array}$ & $\begin{array}{l}626 \mathrm{SM} \\
100 \mathrm{SF}\end{array}$ & \begin{tabular}{|l} 
Divorced, 64\% \\
White. \\
Outcomes not \\
specified by \\
race
\end{tabular} \\
\hline 1999 & Roy & \begin{tabular}{|l} 
Effect of \\
welfare \\
policies on \\
paternal \\
involvement \\
\end{tabular} & $N / A$ & Qual & $40 \mathrm{SF}$ & \begin{tabular}{|l} 
ncludes \\
nonresident \\
fathers. All \\
African \\
American. \\
\end{tabular} \\
\hline 2000 & Brown & $\begin{array}{l}\text { Use of public } \\
\text { monies }\end{array}$ & $N / A$ & $\begin{array}{l}\text { Quant: } 1997 \\
\text { CPS }\end{array}$ & $\begin{array}{l}965 \mathrm{SF} \\
13,065 \mathrm{MF}\end{array}$ & $\begin{array}{l}\text { SF, cohabiting } \\
\text { and not, } \\
\text { widowed, } \\
\text { divorced, } \\
\text { never married } \\
\text { Married } \\
\text { fathers }\end{array}$ \\
\hline
\end{tabular}

Journal of Family Theory \& Review, Vol 7, No. 2 (June 2015): pg. 144-166. DOI. This article is (C) John Wiley \& Sons, Inc. and permission has been granted for this version to appear in e-Publications@Marquette. John Wiley \& Sons, Inc does not grant permission for this article to be further copied/distributed or hosted elsewhere without the express permission from John Wiley \& Sons, Inc. 
NOT THE PUBLISHED VERSION; this is the author's final, peer-reviewed manuscript. The published version may be accessed by following the link in the citation at the bottom of the page.

\begin{tabular}{|c|c|c|c|c|c|c|}
\hline Year & Author(s) & Focus & Theory & Method/ Data & Sample & $\begin{array}{l}\text { Single- } \\
\text { Father } \\
\text { Type(s) }\end{array}$ \\
\hline & & & & & & \begin{tabular}{|l} 
Black, White, \\
Hispanic, but \\
outcomes \\
unspecified by \\
race.
\end{tabular} \\
\hline 2001 & Coles & $\begin{array}{l}\text { Parenting } \\
\text { role identity }\end{array}$ & $N / A$ & Qual & $10 \mathrm{SF}$ & \begin{tabular}{|l} 
Divorced, \\
widowed, \\
never married \\
adoptive. \\
Father only. \\
All African \\
American \\
\end{tabular} \\
\hline 2001 & $\begin{array}{l}\text { Nord \& } \\
\text { West }\end{array}$ & \begin{tabular}{|l} 
Parent \\
involvement \\
in school \& \\
student \\
outcomes
\end{tabular} & $\begin{array}{l}\text { Gender, biological, } \\
\text { family structure, } \\
\text { economic resources }\end{array}$ & \begin{tabular}{|l} 
Quant: 1996 \\
NHES families \\
of Grade $1-12$
\end{tabular} & \begin{tabular}{|l|}
16,145 \\
$2-p$ \\
Stepfamilies \\
SM \\
SF \\
Other
\end{tabular} & $\begin{array}{l}\text { Previous } \\
\text { marital status } \\
\text { not specified. } \\
\text { Race not } \\
\text { specified. }\end{array}$ \\
\hline 2002 & Coles & $\begin{array}{l}\text { Motivations } \\
\text { to parent }\end{array}$ & $\mathrm{N} / \mathrm{A}$ & Qual & $10 \mathrm{SF}$ & \begin{tabular}{|l} 
Divorced, \\
widowed, \\
never married \\
adoptive. \\
Father only. \\
All African \\
American \\
\end{tabular} \\
\hline 2002 & $\begin{array}{l}\text { Hamer \& } \\
\text { Marchioro }\end{array}$ & $\begin{array}{l}\text { Path to } \\
\text { single } \\
\text { fatherhood }\end{array}$ & Ecological & Qual & $24 \mathrm{SF}$ & $\begin{array}{l}\text { Never } \\
\text { married. } \\
\text { All African } \\
\text { American. } \\
\end{array}$ \\
\hline 2004 & \begin{tabular}{||l} 
Battle \& \\
Coates
\end{tabular} & \begin{tabular}{||l}
$\mid$ Child \\
outcomes, \\
school \\
achievement
\end{tabular} & $\mathrm{N} / \mathrm{A}$ & \begin{tabular}{||l} 
Quant: NELS, \\
3 waves
\end{tabular} & \begin{tabular}{|l}
40,907 \\
female \\
students
\end{tabular} & \begin{tabular}{|l} 
Father-only \\
(no other \\
adult). \\
Mother-only. \\
All African \\
American
\end{tabular} \\
\hline 2004 & $\begin{array}{l}\text { Demuth \& } \\
\text { Brown }\end{array}$ & $\begin{array}{l}\text { Adolescent } \\
\text { delinquency }\end{array}$ & Social control & $\begin{array}{l}\text { Quant: } 1995 \\
\text { NLSAH }\end{array}$ & $\begin{array}{l}16,304 \\
\text { adolescents }\end{array}$ & \begin{tabular}{|l}
2 biological \\
married- \\
couple parents \\
SM \& \\
w/stepfather,
\end{tabular} \\
\hline
\end{tabular}

Journal of Family Theory \& Review, Vol 7, No. 2 (June 2015): pg. 144-166. DOI. This article is @ John Wiley \& Sons, Inc. and permission has been granted for this version to appear in e-Publications@Marquette. John Wiley \& Sons, Inc does not grant permission for this article to be further copied/distributed or hosted elsewhere without the express permission from John Wiley \& Sons, Inc. 
NOT THE PUBLISHED VERSION; this is the author's final, peer-reviewed manuscript. The published version may be accessed by following the link in the citation at the bottom of the page.

\begin{tabular}{|c|c|c|c|c|c|c|}
\hline \begin{tabular}{|l} 
Year \\
\end{tabular} & Author(s) & Focus & Theory & Method/ Data & Sample & $\begin{array}{c}\text { Single- } \\
\text { Father } \\
\text { Type(s) }\end{array}$ \\
\hline & & & & & & $\begin{array}{l}\text { SF \& } \\
\text { w/stepmother. } \\
\text { Included } \\
\text { White, Black, } \\
\text { Hispanic, but } \\
\text { outcomes } \\
\text { unspecified by } \\
\text { race. }\end{array}$ \\
\hline \multirow[t]{2}{*}{2004} & \begin{tabular}{|l} 
Zhan \& \\
Pandey
\end{tabular} & \begin{tabular}{|l} 
Education \& \\
economic \\
well-being of \\
single \\
parents
\end{tabular} & Human capital & \begin{tabular}{|l} 
Quant: 1993 \\
PSID
\end{tabular} & $\begin{array}{l}930 \mathrm{SM} \\
168 \mathrm{SF}\end{array}$ & \begin{tabular}{|l} 
Noncohabiting. \\
Race \\
unspecified
\end{tabular} \\
\hline & \begin{tabular}{|l} 
Bokker, \\
Farley, \& \\
Bailey
\end{tabular} & $\begin{array}{l}\text { Father well- } \\
\text { being }\end{array}$ & N/A & Quant & $\begin{array}{l}97 \text { fathers } \\
\text { Midwest }\end{array}$ & \begin{tabular}{|l} 
Divorced fewer \\
than 7 \\
months; full- \\
Custody vs. \\
joint custody \\
vs. \\
noncustody. \\
98\% White \\
\end{tabular} \\
\hline 2006 & Eitle & \begin{tabular}{|l|} 
Adolescent \\
delinquency
\end{tabular} & $\begin{array}{l}\text { Structural, } \\
\text { maternal, paternal, } \\
\text { Same-sex }\end{array}$ & \begin{tabular}{||l}
2002 Florida \\
Youth \\
Substance \\
Abuse, 2000 \\
Census
\end{tabular} & $\begin{array}{l}\text { 9,657 high } \\
\text { schoolers }\end{array}$ & $\begin{array}{l}\text { SF \& SM: 28\% } \\
\text { Black, 13\% } \\
\text { Hispanic, 69\% } \\
\text { White. } \\
\text { Outcomes } \\
\text { specified by } \\
\text { race. }\end{array}$ \\
\hline 2006 & $\begin{array}{l}\text { Hawkins, } \\
\text { Amato, \& } \\
\text { King }\end{array}$ & \begin{tabular}{|l} 
Parental \\
involvement
\end{tabular} & $\begin{array}{l}\text { Gender v. } \\
\text { microstructural }\end{array}$ & \begin{tabular}{|l} 
Quant: 1995 \\
NLSAH
\end{tabular} & $\begin{array}{l}17,330 \\
\text { adolescents }\end{array}$ & \begin{tabular}{|l} 
2-parent \\
married, SM, \\
SF, \\
repartnered \\
(remarried or \\
cohabiting). \\
$70 \%$ White, \\
$14 \%$ Black, \\
$11 \%$ Hispanic. \\
Outcomes not \\
specified by \\
race.
\end{tabular} \\
\hline 2007 & $\begin{array}{l}\text { Hilton \& } \\
\text { Koperafrye }\end{array}$ & \begin{tabular}{|l} 
Parental \\
relations \\
w/extended \\
family
\end{tabular} & $N / A$ & \begin{tabular}{|l} 
Quant: 1992 \\
NSFH
\end{tabular} & $\begin{array}{l}1,792 \mathrm{MM} \\
1,293 \mathrm{MF} \\
916 \mathrm{SM} \\
135 \mathrm{SF}\end{array}$ & $\begin{array}{l}\text { Divorced \& } \\
\text { never married. } \\
75 \% \text { White. } \\
\text { Outcomes } \\
\text { unspecified by } \\
\text { race. }\end{array}$ \\
\hline
\end{tabular}

Journal of Family Theory \& Review, Vol 7, No. 2 (June 2015): pg. 144-166. DOI. This article is (C John Wiley \& Sons, Inc. and permission has been granted for this version to appear in e-Publications@Marquette. John Wiley \& Sons, Inc does not grant permission for this article to be further copied/distributed or hosted elsewhere without the express permission from John Wiley \& Sons, Inc. 
NOT THE PUBLISHED VERSION; this is the author's final, peer-reviewed manuscript. The published version may be accessed by following the link in the citation at the bottom of the page.

\begin{tabular}{|c|c|c|c|c|c|c|}
\hline Year & Author(s) & Focus & Theory & Method/ Data & Sample & $\begin{array}{l}\text { Single- } \\
\text { Father } \\
\text { Type(s) }\end{array}$ \\
\hline 2008 & $\begin{array}{l}\text { Hook \& } \\
\text { Chalasani }\end{array}$ & \begin{tabular}{||l} 
Parental \\
involvement
\end{tabular} & $\begin{array}{l}\text { Microstructural \& } \\
\text { gender }\end{array}$ & \begin{tabular}{|l} 
Quant: 2003- \\
2006 ATUS
\end{tabular} & $\begin{array}{l}7,587 \mathrm{MM} \\
6,184 \mathrm{MF} \\
2,452 \mathrm{SM} \\
431 \mathrm{SF}\end{array}$ & \begin{tabular}{||l} 
Prior marital \\
status \\
unspecified. \\
Majority \\
White. \\
Outcomes not \\
specified by \\
race.
\end{tabular} \\
\hline 2008 & \begin{tabular}{|l} 
Lee \& \\
Kushner
\end{tabular} & \begin{tabular}{||l} 
Children's \\
academic \\
achievement
\end{tabular} & $\begin{array}{l}\text { Same-sex v. } \\
\text { opposite-sex parent } \\
\text { and child }\end{array}$ & $\begin{array}{l}\text { Quant: } 2002 \\
\text { ELS }\end{array}$ & $\begin{array}{l}1,755 \text { high } \\
\text { schoolers }\end{array}$ & \begin{tabular}{|l} 
SF, SM: $61 \%$ \\
White, $10 \%$ \\
Black, $10 \%$ \\
Asian Pacific \\
I slander, 13\% \\
Hispanic. \\
Outcomes \\
unspecified by \\
race.
\end{tabular} \\
\hline 2009 & Coles & \begin{tabular}{|l} 
Father \\
parenting \& \\
well-being
\end{tabular} & $\mathrm{N} / \mathrm{A}$ & Qual & $20 \mathrm{SF}$ & $\begin{array}{l}\text { Father-only. } \\
\text { African } \\
\text { American. } \\
\text { Widowed, } \\
\text { never married, } \\
\text { divorced, } \\
\text { adoption. } \\
\end{array}$ \\
\hline 2009 & \begin{tabular}{|l} 
Forste, \\
Bartkowski, \\
\& J ackson
\end{tabular} & $\begin{array}{l}\text { Father } \\
\text { perceptions }\end{array}$ & Life course & Qual & $\begin{array}{l}36 \text { low- } \\
\text { income } \\
\text { fathers }\end{array}$ & \begin{tabular}{|l} 
Includes \\
nonresident, \\
married, lone, \\
$\&$ cohabiting \\
\end{tabular} \\
\hline 2010 & \begin{tabular}{|l} 
Bronte- \\
Tinkew, \\
Scott, \& \\
Lilja
\end{tabular} & \begin{tabular}{|l} 
Parental \\
involvement \\
$\&$ child \\
outcomes
\end{tabular} & $\begin{array}{l}\text { Life course \& social } \\
\text { capital }\end{array}$ & Quant & $\begin{array}{l}\text { 3,977 youth } \\
\text { NLSY97-3 } \\
\text { waves }\end{array}$ & \begin{tabular}{||l} 
SF, cohabiting \\
and not; SF \\
w/other \\
adults; SM, \\
cohabiting \& \\
not; 2-parent. \\
Black, White, \\
Hispanic but \\
outcomes \\
unspecified \\
\end{tabular} \\
\hline 2011 & Grall & $\begin{array}{l}\text { Child } \\
\text { support }\end{array}$ & $\mathrm{N} / \mathrm{A}$ & $\begin{array}{l}\text { Quant: } 2010 \\
\text { CPS }\end{array}$ & 2010 CPS & \begin{tabular}{|l} 
Custodial \\
fathers \& \\
mothers, could \\
be remarried. \\
Divorced, \\
widowed, \\
never married. \\
$63 \%$ White, \\
$16 \%$ Black, \\
$18 \%$ Hispanic. \\
Racial \\
\end{tabular} \\
\hline
\end{tabular}

Journal of Family Theory \& Review, Vol 7, No. 2 (June 2015): pg. 144-166. DOI. This article is (C) John Wiley \& Sons, Inc. and permission has been granted for this version to appear in e-Publications@Marquette. John Wiley \& Sons, Inc does not grant permission for this article to be further copied/distributed or hosted elsewhere without the express permission from John Wiley \& Sons, Inc. 
NOT THE PUBLISHED VERSION; this is the author's final, peer-reviewed manuscript. The published version may be accessed by following the link in the citation at the bottom of the page.

\begin{tabular}{|c|c|c|c|c|c|c|}
\hline \begin{tabular}{|l} 
Year \\
\end{tabular} & Author(s) & Focus & Theory & Method/ Data & Sample & $\begin{array}{l}\text { Single- } \\
\text { Father } \\
\text { Type(s) }\end{array}$ \\
\hline & & & & & & $\begin{array}{l}\text { outcomes } \\
\text { specified }\end{array}$ \\
\hline 2013 & Livingston & Profile & $\mathrm{N} / \mathrm{A}$ & $\begin{array}{l}\text { Quant: Census } \\
\text { \& IPUMS }\end{array}$ & 2.6 million & \begin{tabular}{|l} 
Divorced, \\
widowed, \\
never \\
married; \\
cohabiting or \\
not, married \\
but separated. \\
Compared to \\
SM and MF. \\
Race specified \\
but \\
descriptive.
\end{tabular} \\
\hline
\end{tabular}

\section{Early Qualitative Research}

As is often the case, the first studies in the 1970s and 1980s were understandably small ( $16-80$ respondents), qualitative, and exploratory, and they were chiefly descriptive and atheoretical. They also largely focused on White, divorced (occasionally widowed), single fathers, who accounted for the majority of single dads in those years. If they included fathers of color, the analysis still often did not address race (this is still largely true today). With few exceptions, such as Ambert (1982), DeFrain and Eirick (1981), Gersick (1979), and Santrock and Warshak (1979), who included groups of single mothers or noncustodial single fathers, these small studies contained no comparison groups.

For the most part, these studies gathered their data from the fathers' perspectives, which is both a strength and a weakness of qualitative research. Such studies give voice to the fathers' perspective, which historically has been missing in most family research, but they are often the voices of select, nonrandomly sampled fathers. In general, the studies found that fathers were capable, confident, and satisfied in their parenting (signifying "well adjusted"), and they reported few behavior problems with their children (Ambert, 1982; Bartz \& Witcher, 1978; Chang \& Deinard, 1980; DeFrain \& Eirick, 1981; Gasser \& Taylor, 1976; Gersick, 1979; 
Orthner, Brown, \& Ferguson, 1976; Orthner \& Lewis, 1979; Risman, $\underline{1986)}$.

Another advantage of qualitative research is that it can more likely focus on the subjective why of a phenomenon, so most of those initial studies investigated the reasons and motivations for father custody and the role of those reasons in fathers' adjustment and satisfaction. Mendes's (1976a) sample of 32 single fathers and Hanson's (1981) study of 37 fathers distinguished between fathers who actively sought custody (seekers) and those who took custody by default (assenters); they found that seekers, despite lacking knowledge of child development, adjusted more easily to single parenthood than did assenters (see also Risman, 1986). Similarly, Gasser and Taylor's (1976) study of 40 single, divorced, and widowed fathers concluded that divorced fathers adjusted better than widowed fathers. In contrast, Rosenthal and Keshet's (1981) 49 full-time single fathers mostly felt that they had had little choice in taking custody of their children; in most cases the mothers had been unable or unwilling. Smith and Smith's (1981) study of 27 single fathers concluded that these men had likely been involved fathers prior to divorce, although Gersick (1979) didn't find the custodial fathers in his study to have been any more involved than his comparison group of noncustodial fathers-nor did Orthner and Lewis's (1979) review of five single-father studies.

The few studies of this period that included a comparison group of single mothers similarly concluded that single-father respondents were doing pretty well-in fact, similar to (DeFrain \& Eirick, 1981) or better than (Ambert, 1982) many single mothers. For instance, Ambert's (1982) single fathers reported better child behavior and higher satisfaction than did single mothers in the study. Ambert attributed this to a "conspiracy" of appreciation; friends and family regularly complimented fathers and offered them more help and social invitations, and that appreciation was reflected to their children, who then also expressed more appreciation of their fathers. Mothers, in contrast, did not receive the same level of kudos and aid, and they reported a lower level of appreciation from their children.

Gersick's (1979) study compared 20 divorced custodial fathers with 20 divorced noncustodial fathers. He found that both sets of men and permission has been granted for this version to appear in e-Publications@Marquette. John Wiley \& Sons, Inc does not grant permission for this article to be further copied/distributed or hosted elsewhere without the express permission from John Wiley \& Sons, Inc. 
had participated in child rearing about equally in their marriages. Instead, what distinguished the two groups was that custodial fathers were more likely middle- or last-born of their siblings, whereas noncustodial fathers were more likely firstborn. Also, custodial men were closer to their mothers and from more traditional gender relations, whereas noncustodial expressed closeness to both parents. (Interestingly, no other studies have followed up on this finding.) Although Gersick suggested that custodial fathers had often been motivated to seek custody because of feeling wronged or betrayed by the wife, he also reported that the vast majority of ex-wives had agreed to father custody. In their review of five single-father studies, Orthner and Lewis (1979) found no evidence that fathers sought custody to deliberately hurt former spouses.

In general, from these studies (and more recent ones), we know that on most key socioeconomic characteristics, single dads lie on a continuum between single moms and married fathers. That is, in terms of income, education, and poverty, single fathers are generally less well-off than married fathers, but they are better off than single mothers. For the most part, this economic pattern has held true throughout the four or five decades that research has been conducted on single fathers (although recently single mothers have surpassed single fathers in education; see Table 1 ). In terms of family configuration, single fathers also consistently differ from single mothers. They tend to have custody of a smaller number of children, older children, and more males than do single mothers (Brown, 2000; Downey, Ainsworth-Darnell, \& Dufur, 1998; Eggebeen, Snyder, \& Manning, 1996; Hook \& Chalasani, 2008; Livingston, 2013; Norton \& Glick, 1986; see also Table 1 ). Also, as Table 1 shows, single fathers are currently less likely than single mothers to have "no other adults" in the household, meaning that they are more likely to have other adults in the household. This is because of their higher rate of cohabitation-they are more than twice as likely as single mothers to cohabit with a partner ( $22.4 \%$ vs. $10.5 \%)$ but less likely to reside with extended family members in complex (i.e., extended) households (21.3\% vs. $29.7 \%)$. not grant permission for this article to be further copied/distributed or hosted elsewhere without the express permission from John Wiley \& Sons, Inc. 


\section{The Turn to Quantitative Research}

Norton and Glick's (1986) demographic study of single fathers using US Census data and Greif and DeMaris's (1990) quantitative study of more than 1,000 single fathers obtained through Parents Without Partners signaled a turning point in research focused on single fathers. Although Greif and DeMaris's respondents were quite homogeneous (96\% White, middle class, and all divorced or separated), there soon followed more systematic studies and growing recognition in the 1990s that single-father homes were increasingly formed by young, never-married men with lower incomes and fewer children (Eggebeen et al., 1996). Since then, the field has been increasingly dominated by quantitative studies using national, more representative data sets. Commonly used data sets include the American Time Use Survey (ATUS), National Education Longitudinal Study (NELS), National Household Education Survey (NHES), National Household Survey on Drug Abuse (NHSDA), National Longitudinal Survey of Youth (NLSY), National Survey of Families and Households (NSFH), National Survey of Family Growth (NSFG), the National Longitudinal Survey of Adolescent Health (NLSAH), and various US Census data (e.g., Current Populations Survey, CPS; Public Use Microdata Sample, PUMS). These better reflect the growing diversity among single fathers, particularly in terms of prior marital status and current living arrangements. Therefore, recent studies more frequently measure within-group variation and test several theories.

\section{Theoretical and Methodological Perspectives on Single Fathering}

In general, studies of single fathers have been interested in two questions: (a) How similarly do single mothers and single fathers parent (sometimes the question is, "Can single dads 'mother'?") (Hook \& Chalasani, 2008; Risman, 1986, 1987), which is frequently measured in terms of time involvement and closeness; and (b) Do the outcomes for children differ by gender of the single parent or by gender matching of the child and parent? I begin with the first question. 


\section{Involvement and Family Processes Among Single Fathers}

Parental involvement is a common focus of parenting studies, and although it is acknowledged that "involvement" is difficult to fully capture, it is often measured in hours or frequency of time(s) spent with children in shared meals, reading, at school, or in leisure activities; by quality of relationship (e.g., respondents' assessment of closeness, attachment, warmth); and investment in the parental role (awareness of and monitoring children's activities, friends, homework). With respect to single-father involvement, scholars have wondered how single fathers' parenting compares to that of married fathers and single mothers. Therefore, researchers have generally tested a microstructural explanation versus a gender explanation. A microstructural approach (sometimes called an interactional approach; see, e.g., Hook \& Chalasani, 2008) argues that fathers and mothers behave differently not because of enduring or inherent gendered traits but because they face different social conditions. Specifically, it is suggested, the social conditions of being a single parent generate similarities in the parenting behaviors of single mothers and single fathers (Nord, Brimhall, \& West, 1997; Risman, 1986, 1987; Thomson, McLanahan, \& Curtin, 1992). The expectations, demands, and opportunities of the single-parenting environment (and from others in that environment, e.g., children) will counter or override gender differences. Therefore, the microstructural approach expects that single mothers and fathers act out gender roles that are less traditionally separate and instead more similar to each other in both their parenting behavior and the amount of time they invest in household work than is the case for mothers and fathers in married two-parent families.

In contrast, a gender approach (sometimes referred to as an individualist approach; see, e.g., Downey et al., 1998) suggests that gender roles, whether manifested in personalities or physiology and whether acquired through biology or early socialization, create stable, immutable parenting attributes. Thus, no matter the parenting situation, men will parent similarly to one another and differently than women. Women will more likely attend to primary and daily parenting 
tasks, such as feeding, dressing, and emotional nurturance of children, whereas men will attend to play activities and financial provision.

Virtually all research finds that single fathers do more household and child-care work when mothers are not around, particularly when they are not in two-parent families. In fact, several studies find that single fathers do more of such work than any other type of father (Cooksey \& Fondell, 1996; Demuth \& Brown, 2004; Hawkins, Amato, $\&$ King, 2006; Nord \& West, 2001; but for an exception, see Jones \& Mosher, 2013). For instance, Nord et al. (1997) used data from the National Household Education Survey to measure involvement in school activities (class events, parent-teacher conferences, volunteering, general school meetings). They found that single mothers and fathers displayed similar rates of participation, with single mothers less involved than married mothers and single fathers more involved than married fathers.

Such findings support a microstructural approach; that is, at least to some extent, men's parenting styles adapt to the contingencies of the new environment. However, most findings have been more mixed. Commonly, studies find evidence, with variation by measure and data set, to support both microstructural and gender theories. The researchers, nevertheless, often conclude that one explanation is stronger than the other.

In her 1987 study of 55 single fathers, 73 single mothers, and 155 married couples, Risman measured role priority, household tasks, child self-disclosure, physical affection, and parent-child intimacy. Single mothers and fathers were similar on most measures, but single mothers reported more physical affection and intimacy with their children than did single fathers. Risman concluded that microstructural elements were more important explanatory factors than was gender. (Similarly, see Demuth \& Brown, 2004; Heath \& Orthner, 1999; Hilton \& Devall, 1998; Risman, 1986, 1987; Thomson et al., 1992.)

However, Hawkins et al. (2006) analyzed adolescent reports from the 1995 NLSAH to compare parental involvement among twoparent, single-parent, and stepparent households, as well as nonresident parents. Using 10 measures of involvement (shopping, playing sports, religious and cultural events, school projects, various 
types of verbal interaction, and closeness), the researchers found that teens from single-father households rated their fathers highest among all fathers, supporting a microstructural view. Nevertheless, single fathers were no more involved overall than nonresident mothers, and unpartnered single mothers rated higher on involvement than single dads on all 10 measures. Hence, the authors concluded that the results supported a gender approach, as gender accounted for approximately $95 \%$ of the variance between parental categories.

Using NSFH 1987-1988 data, Hall, Walker, and Acock (1995) compared noncohabiting single-mother $(1,433)$ and single-father (128) households. They found that single mothers and fathers did not differ in the number of meals per week they ate with at least one of their children, and they spent similar amounts of time with children in other activities, such as doing homework or reading. Thus, these findings provided some support for microstructural theory. But Hall et al. also found that single fathers spent slightly more time than single mothers in leisure activities with children away from home, and mothers spent slightly more time in private talks with children. More significant, parents differed considerably in the time spent in housework; single mothers spent more time on housework overall and more time specifically on "feminine" tasks (e.g., meal preparation, washing dishes, cleaning, laundry), whereas single dads spent more time on "masculine" tasks (e.g., outdoor work, auto maintenance), indicating that household duties remained gendered.

Hook and Chalasani (2008) approached their study from a more nuanced perspective. The authors suggested that not all interactional (microstructural) pressures encourage men to behave as mothers; single fathers face competing pressures to behave both as moms and as traditional men. They argued that the "doing gender" perspective suggests that, regardless of structural position, gender exerts distinct pressures holding individuals accountable to their gender. They argued that even in identical structural positions behaviors can be expected to diverge by gender precisely because the microstructural position is incongruent with macro gender norms. The ideology of intensive motherhood, for example, shapes the involvement of mothers even if they are noncustodial, and the "good provider" cultural norm pressures single fathers to forefront provision even when they are primary caretakers.

Journal of Family Theory \& Review, Vol 7, No. 2 (June 2015): pg. 144-166. DOI. This article is @ John Wiley \& Sons, Inc. and permission has been granted for this version to appear in e-Publications@Marquette. John Wiley \& Sons, Inc does not grant permission for this article to be further copied/distributed or hosted elsewhere without the express permission from John Wiley \& Sons, Inc. 
With this in mind, using 2003-2006 ATUS data, Hook and Chalasani (2008) compared single fathers to married fathers and single mothers in terms of the amount of time spent on child care, accounting for differences in employment profiles, household composition, and care arrangements. They found that single fathers spend slightly less time caring for children than single mothers, but more time than married fathers. In particular, for children age 5 years and younger, single fathers spend less time on physical care and more time on play than do single mothers. Single fathers spend less time on housework and more time eating. The researchers concluded that both microstructural and gender theories were supported.

Where studies have been most consistent are those measuring closeness, monitoring, and supervision; these have been most likely to find that single fathers are less close to and less involved with their children's friends and school, and monitor and supervise their children less than single mothers do (Bronte-Tinkew, Scott, \& Lilja, 2010; Buchanan, Maccoby, \& Dornbusch, 1996; Cookston, 1999; Demuth \& Brown, 2004; Downey, 1994; Hall et al., 1995; Hilton \& Devall, 1998; Maccoby \& Mnooken, 1992; Risman, 1987).

For instance, with 1995 NLSAH data on 7th-12th graders, Cookston (1999) used three measures of parental supervision: how often the parent is home when the child leaves for school, when the child returns, and when the child goes to bed. Compared to single mothers and two-parent families, single fathers had the lowest supervision scores. However, Cookston pointed out, as did Hook and Chalasani (2008), that because single fathers have greater employment hours than single mothers and married fathers, their employment may account for their lower supervision.

Finally, using their own data set of 30 each of single fathers, single mothers, and married couples, Hilton and Devall (1998) measured 18 positive (e.g., spending time together, comforting, showing affection) and negative (e.g., criticizing, yelling, nagging) parental behaviors through a survey administered to both parents and children. They found that single fathers displayed more positive behaviors than married fathers. But similar to the already-mentioned studies, they concluded that single fathers were less restrictive than single mothers; they were more likely to allow their children to and permission has been granted for this version to appear in e-Publications@Marquette. John Wiley \& Sons, Inc does not grant permission for this article to be further copied/distributed or hosted elsewhere without the express permission from John Wiley \& Sons, Inc. 
participate in the activities of their peers and try activities on their own.

\section{Within-Group Variation}

Descriptive in nature, Brown's 2000 study, based on 1997 CPS data, began laying out the demography of within-group variation. Brown compared married fathers to single fathers, but he also divided the latter into nine types by previous marital status (divorced, widowed, and never married) and by current living arrangements (cohabiting; lone; or complex, i.e., living with extended family) to delineate demographic differences. Among single fathers, Brown found that cohabiting fathers (about $25 \%$ of single fathers) had the least amount of education and income and were younger than married or noncohabiting fathers. Cohabiting fathers were more likely to be racial minorities and least likely to be married. Sixteen percent of Black fathers were single (twice the overall rate). Never-married fertility was the primary path for cohabiting single fathers (61\% had never been married), but divorce was the primary path for noncohabiting fathers, although $25 \%$ of all noncohabiting fathers were never married. More than $33 \%$ of cohabiting fathers were divorced or separated, and so probably not living with the child's mother. The vast majority of single fathers headed their own household; about 14\% of noncohabiting fathers lived in households headed by his parent or other adult (compared to $6 \%$ of cohabiting single fathers and $2 \%$ of married fathers). Most (60\%) single fathers lived with only one child. Thirtyfour percent of cohabiting single fathers had young children (younger than age 2 ), whereas only $10 \%$ of noncohabiting single fathers and $19 \%$ of married fathers had children that young.

Related to parenting involvement, the few within-group variation studies have focused on comparing noncohabiting to cohabiting single fathers. Bronte-Tinkew et al. (2010) used two theoretical perspectives: (a) a life course perspective that suggests that recent and past experiences, as well as interactions and relationships with family members, contribute to current conditions and roles (Elder, Liker, \& Cross, 1984; Roberts \& Bengston, 1993), and (b) a social capital framework, suggesting that the strength of ties and levels of closeness and involvement between single fathers and 
their adolescent offspring would aid adolescents in future development of financial and human capital. Specifically, Bronte-Tinkew et al., using five rounds of NLSY data from 1997 to 2003 and using only those cases in which the family structure remained the same throughout the five rounds, explored how the presence of cohabiting partners affects parenting involvement among single fathers. The researchers found that single custodial-father families with a coresident partner had the lowest levels of family routines; adolescents in such families are least likely to participate in regular family activities such as eating dinner together. Single-father households with a partner also exhibited lower levels of closeness and awareness of their children's friends and activities than all other parent types, which may lower social capital for the child.

Similarly, the previously mentioned Hawkins et al. (2006) study found that unpartnered single fathers had the highest levels of engagement with their adolescent children in comparison to any other type of father, particularly in regard to traditionally feminine activities. These findings confirmed a few earlier studies (Maccoby \& Mnookin, 1992; Thomson et al., 1992) that also found that parental involvement tends to be lower in cohabiting families relative to both single-parent families and married two-parent families.

\section{Outcomes for Children of Single Fathers}

The discussion of parenting differences between single mothers and fathers flows logically into a discussion of the second question addressed in many studies of single fathers: whether parenting differences, even modest ones, between single mothers and single fathers have important consequences for children's well-being. If not, this gives greater credence to the microstructuralist view. If significant outcome differences exist, then individualist, gendered arguments carry more weight.

For several decades, many studies focused on single-mother households, comparing their children's outcomes to those of twoparent families. Most found that living in a single-mother family increases the risk for delinquency. In those studies, the most popular explanation centers on a resource deprivation argument-single mothers have fewer resources (e.g., income, time, energy), thus 
leading to diminished social control and socialization (Amato \& Keith, 1991; Lareau, 1989; McLanahan \& Booth, 1989; McNulty \& Bellair, 2003; Meyer \& Garasky, 1993; Rankin \& Wells, 1994). Because we now know that, on average, single fathers have higher income and, until recently, higher education than single mothers, and fewer children in whom to invest those resources, we might expect that single fathers would be more effective than single mothers at controlling child delinquency.

To explore this expectation, several studies have tested what Eitle (2006) called the "maternal" and "paternal" hypotheses, although the studies do not always explicitly refer to those hypotheses. The maternal hypothesis suggests that children are less likely to be engaged in delinquency when raised by single mothers, instead of single fathers because single fathers have weaker interpersonal and affective bonds with their children. This thesis often assumes that men also have less access to extended family to buttress social control, but according to Hill and Hilton (1999) and Hilton and Koperafrye (2007), single fathers have as much, if not more, access to support from friends and extended families. In contrast, the paternal hypothesis suggests that children of single fathers are at lower risk because men are more effective disciplinarians, but, as mentioned already, several studies indicate that single fathers exhibit less closeness, supervision, and monitoring, and so we should not expect much support for this thesis. As explained before, a structuralist theory suggests that single fathers and mothers adapt to the exigencies of the single-parent context, which makes their children at about the same risk for delinquent or "deviant" behavior.

Using data from the 1982 NSFG, McLanahan and Bumpass (1988) found no differences in the likelihood of teen marriage, teen birth, premarital birth, or marital disruption between youths in singlemother households and youths in single-father households. Adult children from both single-father and single-mother households had equivalently higher rates of these outcomes than those in two-parent households.

Most more recent studies have concentrated on adolescent respondents and have distinguished between internalizing behaviors (e.g., depression, anxiety, low self-esteem) and externalizing and permission has been granted for this version to appear in e-Publications@Marquette. John Wiley \& Sons, Inc does not grant permission for this article to be further copied/distributed or hosted elsewhere without the express permission from John Wiley \& Sons, Inc. 
behaviors (e.g., antisocial or violent behavior) and substance use. Current evidence from these studies indicates that for internalizing behaviors (Buchanan et al., 1996; Downey et al., 1998) and academic performance (Downey, 1994; McLanahan \& Sandefur, 1997; Mulkey, Crain, \& Harrington, 1992), outcomes for children from single-father and single-mother households are similar. Again these conclusions support a microstructural approach.

However, turning the lens to externalizing behavior (e.g., antisocial and violent behavior) and substance use (e.g., cigarette smoking, alcohol, drugs), parental gender effects become more salient, with children of single fathers consistently showing higher levels of both (Buchanan et al., 1996; Cookston, 1999; Demuth \& Brown, 2004; Downey \& Powell, 1993: Hoffmann \& Johnson, 1998) over children of single mothers. (Although this review is of US studies, I note that Breivik and Olweus's (2006) study of Norwegian single fathers came to the same conclusions.)

For instance, Eitle's own findings from the 2006 study using data from the Florida Youth Substance Abuse Survey, an annual survey of middle and high school students, found that living with a single father increased the risk of alcohol use among boys and girls, the risk of delinquent behavior among daughters, and the risk of marijuana use among Latino students (the study included Whites and African Americans as well). Parental gender didn't matter for other illicit drug use. The inconsistent nature of the findings led Eitle (2006) to conclude that both the microstructural and the maternal hypotheses were somewhat supported, but not the paternal hypothesis.

Hoffmann and Johnson (1998) focused on drug use among adolescents ages 12-17, using 3 years of NHSDA data. They compared family structures that included two parents, single parents, and stepparents, and they concluded that the risk of drug use, including problem use, was greatest for adolescents in single-father households (see also Cooksey \& Fondell, 1996). Similarly, Cookston (1999) used 1995 NLSAH data (adolescent reports) to measure involvement (parental supervision) and outcomes. He found that alcohol and drug behaviors, as well as delinquency rates, were highest in single-father homes. Using the same data, Demuth and Brown (2004) likewise found that family process scores (measures of closeness, supervision, 
and monitoring) were consistently higher in single-mother families, and this was reflected in lower delinquency rates among children of single mothers versus those of single fathers. However, once they controlled for family process variables-that is, once they compared single mothers and fathers with similar levels of closeness, attachment, supervision, and monitoring-they concluded that gender was of no importance.

One of the few exceptions was a study conducted by Downey, Ainsworth-Darnell, and Dufur (1998). Using 1990 NELS data, which includes reports from adolescent students, parents, and teachers, Downey et al. compared adolescents from single-mother and singlefather households on adolescent well-being (measured as teen parenthood, delinquency, marijuana use, disruptive behavior, getting along with others, and effort in school). Only two measures revealed significance-teachers judged youths raised in a single-father household as less successful at getting along with others and putting forth effort. There were no significant differences on self-concept or relationships with peers. Controlling for socioeconomic resources, children from single-father families had slightly lower standardized test scores. However, given the small size and number of differences, the researchers concluded that there was more support for a microstructuralist view. If women's and men's contributions are distinctive, they would have expected larger and more consistent differences in adolescent outcomes that those observed. In addition, Clarke-Stewart and Hayward's (1996) study found that children were emotionally better off in single-father homes, but they were studying younger children (ages 5-13) and were not using a national, representative sample.

Related to outcomes and within-group variation, Buchanan et al.'s (1996) Stanford Custody Project found that having a cohabiting partner in the household, which as stated earlier is more common among single fathers than single mothers, was associated with higher levels of virtually every problematic outcome they measured: poorer conflict resolution skills, substance use, school deviance, antisocial behavior, and lower grades and effort at school. Not surprisingly, the authors concluded that the association between having an unmarried partner in the household and poor adjustment, especially for boys, was strong and consistent.

Journal of Family Theory \& Review, Vol 7, No. 2 (June 2015): pg. 144-166. DOI. This article is @ John Wiley \& Sons, Inc. and permission has been granted for this version to appear in e-Publications@Marquette. John Wiley \& Sons, Inc does not grant permission for this article to be further copied/distributed or hosted elsewhere without the express permission from John Wiley \& Sons, Inc. 


\section{Interaction Between Sex of Parent and Child}

Several studies related to outcomes for children have gone one step further than asking whether children are better off with single fathers or single mothers; rather, they have asked whether the consequences for children's outcomes are a result of an interaction between the gender of the parent and of the child. For the most part, these studies ask whether children will fare better when they are raised by a parent of the same sex. Remember that single fathers tend to raise more sons than daughters, which can be attributed to both a greater propensity of fathers to seek custody of their sons and to mothers' and courts' willingness to grant those requests, in part because the parties assume that fathers will be more effective parents for sons than for daughters. Underlying this is Freud's classic psychoanalytic theory, which emphasizes the importance of a child's ability to identify with the same-sex parent as a prerequisite for his or her healthy emotional development (Downey \& Powell, 1993). Similarly, social learning theory stresses the importance of the child modeling the behavior of the parent more similar to her- or himself, as well as the reinforcement received from others for doing so (Bussey \& Bandura, 1984). In addition, others have suggested that parents may better understand the needs of their same-sex children (Thompson, 1983), or researchers have highlighted concerns that custodial heterosexual parents may seek emotional fulfillment from their opposite-sex children in the absence of an adult partner (Weiss, 1979).

Among the first studies to support the same-sex theory was the Texas Custody Research Project (Santrock \& Warshak, 1979), which compared 64 White middle-class families, matched on socioeconomic status, size, and sibling status. About 33\% were intact families, 33\% mother custody, and 33\% father custody (all were single parents following divorce). The children were compared on several psychological well-being measures (e.g., self-esteem, warmth, anxiety, anger, maturity, sociability, conformity, independence). The results of the study, published in several articles (see, e.g., Santrock \& Warshak, 1979; Warshak \& Santrock, 1983), concluded that the pattern of results consistently revealed more socially competent behavior in children living with the same-sex parent than in children 
living with the opposite-sex parent. Boys in father-custody homes showed greater maturity and sociability than did girls in father-custody homes, and girls in mother-custody homes were rated as more socially competent than boys in mother-custody homes. In addition, children were more likely to indicate a preference for same-sex custody.

However, subsequent studies have not reached the same conclusions. Using data from the 1988 NELS, Downey and Powell (1993) looked at 35 social, psychological, and educational outcomes and could not find even one in which both males and females benefit significantly from living with their same-sex parent. Only four outcomes regarding interaction between the sex of the parent and the child were significant, and the effects were counter to the same-sex thesis: On several educational outcomes-educational expectations of the child, some standardized test scores, and educational objects in the home-girls scored higher if they were in father-only households.

Using a sample of 187 children from 160 divorced families in Southern California, with roughly equal numbers of children in samesex and opposite-sex custodial arrangements, Clarke-Stewart and Hayward (1996) tested the maternal versus paternal theory, hypothesizing that children would do better in the custody of their same-sex parent. Although they found that children were generally emotionally better off in father custody, none of the interactions by gender matching of child and parent was significant for any measure of psychological well-being (e.g., divorce adjustment, self-esteem, depression, anxiety). (A 1998 study in I srael by Guttman and Lazar came to similar conclusions.)

\section{Summary}

In summary, what can we conclude regarding single fathers and their children? Single fathers tend to be better off in terms of income and social support than single mothers. They tend to have fewer, older, and more male children than single mothers. More often than not, studies indicate that single fathers' involvement with children and household duties increases as they take on the single-parent role. This involvement is quantitatively similar to that of single mothers but perhaps differs in traditionally gendered ways, with single mothers being more likely to participate in private talks and housework, and permission has been granted for this version to appear in e-Publications@Marquette. John Wiley \& Sons, Inc does not grant permission for this article to be further copied/distributed or hosted elsewhere without the express permission from John Wiley \& Sons, Inc. 
whereas single fathers are more involved in play and provision. Single mothers tend to provide more closeness, monitoring, and supervision than do fathers, who appear more lenient, allowing children to experiment a bit more. Thus, these studies provide support for both microstructural (single dads are more involved than married dads) and gender theories (single dads' and single moms' parenting nevertheless differs in some qualitatively gender-normed ways).

With a few possible exceptions, the children of single fathers do about as well in terms of internalizing behavior and academic performance (sometimes better), which again provides support for microstructural theories. However, the children of single fathers appear to be more likely to participate in externalizing behavior and substance use (do not confuse with "abuse"), perhaps a reflection of the already-mentioned style differences, which indicates that resources play a lesser role than parental processes in these outcomes and provides some support for maternal theories. As of yet, the few studies of young adults (as opposed to adolescents) do not seem to indicate significant long-term differences, as related to marriage, teen birth, and divorce, between those reared in single-father versus singlemother homes (Downey \& Powell, 1993; McLanahan \& Bumpass, 1988).

\section{Discussion}

Early research on single mothers often generalized findings to all single parents (e.g., Bianchi, 1995). So the inclusion of, or focus on, single fathers has contributed to a resolution of that bias. Nevertheless, further refinements are needed within the single-father literature itself. Hill and Hilton (1999) complained that the literature on single fathers suffered from poorly defined samples, thus making comparison across studies difficult. To some extent, that weakness lingers and is difficult to resolve. As mentioned earlier, getting a firm grasp of the prevalence of single fathers in the United States can be slippery because single fathers can be called by various labels with slightly different meanings-for example, father-only household or lone father usually means that the child's mother is not present, but another partner might be. In some studies, single fathers includes fathers who are nonresident with their children (Forste, Bartkowski, \& 
Jackson, 2009; Roy, 1999). In addition, custodial fathers can include remarried fathers who have custody of their children (see Grall's 2011 study on receipt of child support). Some researchers may try to delete remarried custodial or cohabiting single fathers from their studies, but it often depends on the focal research question as to whether that is advisable. In addition, the definition of children may vary. Most commonly included are households containing children younger than age 18 , but occasionally studies include children younger than age 21 , which, of course, increases the overall count of single fathers. Hence, "single fathers" is a fluid concept, and it pays to read the fine print.

Once the pool has been defined, some researchers fail to distinguish prior marital status-never married, divorced or separated, and widowed (although most studies continue to focus on divorced fathers). The different transitions involved in these may portend very different outcomes for father and child. I have seen no studies on single fathers that distinguish among biological or adoptive fathers. Some studies exclude fathers who are living with extended kin (complex households), whereas others allow those fathers to be among the respondents but may not control for that. Controlling for those distinctions is essential, because demographic studies indicate that fathers differ greatly in income, race, education, and age, according to marital status and current living arrangements. Finally, although we can assume that inevitably some of the single fathers are gay, none of the studies using large data sets distinguish sexual orientation.

For at least the first two decades, virtually all studies on single fathers focused on White fathers. Although that has changed with the use of nationally representative data sets, most studies, except those profiling demographic differences among single fathers (e.g., Brown, 2000; Eggebeen et al., 1996; Grall, 2011; Meyer \& Garasky, 1993), do not delineate findings according to race. (Among the few exceptions, see Eitle, 2006; Zhan \& Pandey, 2004; the research of the latter, not described herein, looked at the effect of a 4-year college education on economic well-being of single parents.) Although research on fatherhood among various racial groups is increasing, the status of studies on single fathers of color currently resembles those of White single fathers of the 1970s and 1980s; that is, the studies are small, qualitative, descriptive, and exploratory in nature, and they focus on

Journal of Family Theory \& Review, Vol 7, No. 2 (June 2015): pg. 144-166. DOI. This article is (C) John Wiley \& Sons, Inc. and permission has been granted for this version to appear in e-Publications@Marquette. John Wiley \& Sons, Inc does not grant permission for this article to be further copied/distributed or hosted elsewhere without the express permission from John Wiley \& Sons, Inc. 
African American single fathers (Coles, 2009; Green, 2010; Hamer \& Marchioro, 2002). Battle and Coates (2004) and Battle and Scott (2000) are the only larger, comparative, quantitative studies on outcomes of Black children in single-father homes. I have seen no studies focused on single fathers of other ethnic minority groups.

Most research on single fathers, whether qualitative or quantitative, has been cross-sectional in nature, thus prohibiting the examination of cause and effect. Even research using longitudinal data sets often uses only one wave of data in the study (Bronte-Tinkew et al., 2010, is an exception). In addition, cross-sectional data make it difficult, if not impossible, to rule out selection effects of the children and the father. For instance, in terms of higher delinquency rates, does single fathering precede delinquency of children, or vice versa? That is, is there a tendency to place problematic children with male parents? Although Powell and Downey (1997) could find no evidence of more troubled children, particularly boys, being sent to live with their fathers, they came to this conclusion by determining that a very small percentage of the children in their study had switched from living with single mothers to living with single fathers. They were unable to determine whether more troublesome children had gone to live with fathers immediately at the initial divorce. Most existing studies cannot attempt to address that question.

Single fathers do have more choice (seekers) than single mothers regarding whether and when they assume child custody, and some studies have found that father custody is more likely to be the result of a problematic family environment (e.g., ex-spouse or mother has serious emotional problems, high interparental conflict) than when mothers obtain custody (Buchanan et al., 1996). So children's outcomes, whether positive or negative, could to some extent be selection effects of the single father or the child. More longitudinal data would be able to address these questions better.

Several important variables have no measure in most studies, and thus researchers are unable to account for their effects. These include the following: 
- Length of time children have been living in a single-father household (exceptions: Bokker, Farley, \& Bailey, 2006, whose respondents had to be divorced fewer than 7 months).

- Extent of involvement of any noncustodial parents. Hawkins et al. (2006) and Nord and West (2001) found that nonresident mothers were more involved than nonresident fathers. ClarkeStewart and Hayward's (1996) conclusions were less clear. They found that nonresident mothers and fathers were no different "in the amount or kind of contact they had with their children (frequency of visits, length of visits, shared activities, and holidays). Nor did nonresident mothers live closer to their children" ( p. 263), but they also said that nonresident mothers "somehow seemed to stay more involved with their children" (p. 263) and that children living with their fathers were more likely to continue thinking of their mothers as part of the family and in more positive terms than did children in mother custody of their nonresident fathers. More research on this might clear up the role of nonresident parents.

- Measures of income or poverty (these are more common in studies but still not present across the board).

- Number of children (most usually use one focal child), types of custody (split, joint, sole), whether the father also has custody of nonbiological children or has other biological but nonresident children.

- Hours employed by parents. As mentioned earlier, the studies that did include hours employed (Hook \& Chalasani, 2008; Lin \& Chen, 2006) consistently found that single fathers spend more time in the paid labor force, so this would seem to be an important variable to include on a consistent basis.

Most of the child outcome research has been conducted on teens. The field of single-father research would be enhanced by more studies on adult children of single fathers. This would provide insight into whether those higher rates of substance use, for instance, continue into adulthood and whether they convert to higher levels of abuse. In what ways do the lower levels of talk, affection, and closeness apparently associated with single fathering translate into adult childfather relations or into the relationships the adult child creates with his or her peers and partners?

Although the shift to quantitative studies has contributed to the quality of the research in several ways (broadening the pool to be more representative; containing respondent numbers large enough to 
make generalizations; controlling for factors that qualitative studies could not capture; including respondents other than the fathers themselves (e.g., mother, children, teachers), many quantitative studies are less focused on the single father and his family per se and more on comparing the effects of various family structures (e.g., married, step, cohabiting, single), among which single fathers happen to be a small subset. Consequently, there is still room for good qualitative studies to focus in depth on the experience, motivations, and perceptions of single fathers and their children, asking how the children themselves play a role in shaping the fathering experience and expanding to special subgroups (e.g., different racial groups, sexual orientation, adoptive fathers), for which exploratory, descriptive studies may be most useful.

\section{Conclusion}

In terms of theoretical directions, the research has understandably fallen into a dichotomous comparison of motherfemale-feminine versus father-male-masculine parenting, often with the goal of determining who is the better parent (and often with the presumption that "mothering" is the gold standard, as illustrated in Risman's, 1986, “Can Men Mother?"). When single-father parenting seems to be more similar to that of a single mother's and less similar to that of a married father's, more weight is given to a microstructural explanation, even when we know little about the structural situation itself, except that it has one fewer parent. A microstructural hypothesis would best be tested by a longitudinal study that follows the same men who are first in a married or two-parent situation and who then subsequently enter a single-parent situation. However, as we all know, it is hard to create perfect social experiments with human lives. Though still imperfect, qualitative studies could partially capture these transitional structural effects on fathers' parenting goals, styles, and outcomes by purposively sampling fathers who have experienced this transition, but such studies would still have the limitation of retrospective answers.

The preponderance of evidence indicates that both microstructural factors and gender are at play. Single fathers parent differently than married fathers because of the needs of the structural 
context, particularly the number of adults available. But by the time they reach single fatherhood, fathers have already spent a lifetime in their genes and gendered norms, and the effects of these pervasive normative factors likely resist total obliteration. Hence, in this respect, it is probably time to move the research on single fathers in a more integrated direction.

First, similar to the model used in Hooks and Chalasani (2008), theory should assume that parenting reflects the constraints and pressures associated with both gender and structural perspectives. Which constraints and pressures do fathers (and mothers) perceive regarding their gendered roles as men (and women) and as fathers and mothers? For instance, do single fathers feel more pressured to be employed, to be providers, to take off less work time, than do single mothers? In what ways and to what extent do single fathers hold themselves to lower or higher parenting standards than do single mothers? How do such differences in gendered expectations, if any, play out in their parenting in practical ways? Because of gender differences and gendered cultural expectations, fathers appear to have more choice about whether and when they take custody of children; to what extent are father and child outcomes influenced by selection factors mentioned earlier? Are single fathers more motivated to parent than single mothers? Research already indicates that single fathers are more financially prepared, but does this extend to other resources, such as social and emotional?

Second, despite having just suggested ways that research could compare gendered roles within a structural model, a second line of research needs to veer from the research comparing mothers and fathers and continue the nascent trend toward more within-group variation research, using more ecological, family systems, or developmental models, for instance. More studies that make comparisons among the single fathers themselves-such as between cohabiting and noncohabiting fathers or between those fathers living or not living with relatives, will inform us about the dynamics and effects of various coparenting configurations. Studies distinguishing among fathers of previous marital statuses (e.g., divorce, widowhood, never married) will help us better understand the preparation and transition effects of life passages. Sorely needed are studies comparing adoptive and biological fathers or gay or straight fathers or fathers by and permission has been granted for this version to appear in e-Publications@Marquette. John Wiley \& Sons, Inc does not grant permission for this article to be further copied/distributed or hosted elsewhere without the express permission from John Wiley \& Sons, Inc. 
race. A growing population, though still small, is men who have children by more than one woman, which adds at least another layer of structural constraints and pressures; an exploratory study on this population could be quite useful to scholars, policymakers, and practitioners alike, at least those who want to make policy and practice evidence based. Studies such as these would fill a huge chasm in the literature on single fathers.

Third, as mentioned earlier, most studies on outcomes have looked at middle school and high school children. More studies focusing on the adult population, where the long-term results can be detected, are essential. In this respect, studies that go beyond the "child's" educational attainment, school behavior, and the like, to look at attitudinal differences, their relationships with their parents, and the effects on their own life choices and parenting would greatly facilitate the evaluation of single fatherhood.

Finally, although the recent increase in single fatherhood has sparked a welcome upsurge in research on single fathers, there are still more than five times as many children $(23.7 \%)$ residing in mother-only than in father-only households (4.1\%) as of 2013 (see Table 1 ). The percentage of children living with neither parent has always been higher than those living with fathers only and has also risen since the 1970s (Kreider \& Ellis, 2011). This could indicate that there is room for growth in single-father households, although as mentioned earlier, census data since 1880 indicate that the percentage of children residing in father-only families has never hit $5 \%$ (Kreider \& Ellis, 2011), which raises the question as to whether single fathering has a "glass ceiling." From a practitioner perspective, teachers, social service providers, local community providers, and the courts could continue to (or increase their efforts to) facilitate father responsibility and inclusion in the family matrix. Even when fathers are not custodial parents, including them-by communicating with them directly, not through the mother or child-in school activities and social services will help prepare men who might end up being primary custodial parents in the future. Increasing the employment of men in social services and local community centers also increases the idea that men can be proficient caretakers. Although single fathers are often financially better resourced than single mothers, there are many who could use social services. Fathers need to be better apprised of social and 
community services available to them and access to such services needs to be facilitated. Homeless shelters, to give an example of more dire circumstances, often allow only single men or mothers with children, not men with children. The foregoing studies indicate that single fathers give their children more freedom, with the result that adolescents of single fathers tend to experiment more with drugs and alcohol. We don't know whether this carries into adulthood or results in long-term substance abuse, but practitioners who work with children of single fathers could incorporate this information into their parenting programs and counseling.

Rather than researchers and practitioners keeping score as to who makes the best parent, or posing differences between single mothers and fathers as deficits, research could focus on identifying the obstacles to single fatherhood, as well as on the potential strengths of single fathering (or male parenting generally) and how those can be replicated. In the long-term such a strategy could contribute to a closing of the gap in the percentage of children living with singlefathers as compared to single mothers and to more cooperative coparenting.

\section{* References marked with an asterisk indicate studies included in the systematic review.}

Amato, P. R., \& Keith, B. (1991). Parental divorce and the well-being of children: A meta-analysis. Psychological Bulletin, 110, 26-46.

*Ambert, A. (1982). Differences in children's behavior toward custodial mothers and custodial fathers. Journal of Marriage and Family, 44, 7386.

Bartz, K. W., \& Witcher, W. C. (1978). When father gets custody. Children Today, 7, 2-6, 35.

Battle, J., \& Scott, B. (2000). Mother-only versus father-only households: Educational outcomes for Black men. Journal of Black Men, 5, 93-116.

*Battle, J ., \& Coates, D. L. (2004). Father-only and mother-only, singleparent family status of Black girls $\&$ achievement in grade 12 and at two years post high school. Journal of Negro Education, 73, 392-407.

Bianchi, S. M. (1995). The changing demographic and socioeconomic characteristics of single parent families. Marriage and Family Review, 20, 71-97. 
*Bokker, L. P., Farley, R. C., \& Bailey, W. (2006). The relationship between custodial status and emotional well-being among recently divorced fathers. J ournal of Divorce \& Remarriage, 44, 83-98.

Breivik, K., \& Olweus, D. (2006). Adolescents' adjustment in four post-divorce family structures. J ournal of Divorce \& Remarriage, 44, 99- 124.

*Bronte-Tinkew, J., Scott, M. E., \& Lilja, E. (2010). Single custodial fathers' involvement and parenting: Implications for outcomes in emerging adulthood. J ournal of Marriage and Family, 72, 1107-1127.

*Brown, B. V. (2000). The single-father family: Demographic, economic, and public transfer use characteristics. Marriage and Family Review, 29, 203- 220.

Buchanan, C. M., Maccoby, E. E., \& Dornbusch, S. M. (1992). Adolescents after divorce. Cambridge, MA: Harvard University Press.

Bussey, K., \& Bandura, A. (1984). Influence of gender constancy and social power on sex-linked modeling. Journal of Personality and Social Psychology, 47, 1292-1302.

*Chang, P. N., \& Deinard, A. S. (1982). Single-father caretakers: Demographic characteristics and adjustment processes. American Journal of Orthopsychiatry, 52, 236-243.

Child Trends Databank. (2013). Births to unmarried women. Retrieved from http://www. childtrends.org/?indicators=births-to-unmarried-women

* Clarke-Stewart, K. A., \& Hayward, C. (1996). Advantages of father custody and contact for the psychological well-being of school-age children. Journal of Applied Developmental Psychology, 17, 239-270.

*Coles, R. L. (2001). The parenting roles and goals of single Black full-time fathers. Western J ournal of Black Studies, 25, 101-116.

*Coles, R. L. (2002). Black single fathers: Choosing to parent full-time. Journal of Contemporary Ethnography, 31, 411-439.

*Coles, R. L. (2009). Best kept secret: Single Black fathers. New York, NY: Rowman \& Littlefield.

*Cooksey, E. C., \& Fondell, M. M. (1996). Spending time with his kids: Effects of family structure on fathers' and children's lives. Journal of Marriage and the Family, 58, 693-707.

*Cookston, J. T. (1999). Parental supervision and family structure: Effects on adolescent problem behaviors. Journal of Divorce \& Remarriage, 32, 107- 122.

*DeFrain, J., \& Eirick, R. (1981). Coping as a divorced single parent: A comparative study of fathers and mothers. Family Relations, 30, 265273.

*Demuth, S., \& Brown, S. L. (2004). Family structure, family processes, and adolescent delinquency: The significance of parental absence versus parental gender. Journal of Research in Crime and Delinquency, 41, 58-81. 
* Downey, D. B. (1994). The school performance of children from singlemother and single-father families: Economic or interpersonal deprivation? Journal of Family Issues, 15, 129-147.

* Downey, D. B., Ainsworth-Darnell, J. W., \& Dufur, M. J. (1998). Sex of parent and children's well-being in single-parent households. Journal of Marriage and Family, 60, 878-893.

* Downey, D. B., \& Powell, B. (1993). Do children in single-parent households fare better living with same-sex parents? Journal of Marriage and Family, 55, 55-71.

* Eggebeen, D. J., Snyder, A. R., \& Manning, W. D. (1996). Children in singlefather families in demographic perspective. Journal of Family Issues, 1, 441-465.

*Eitle, D. (2006). Parental gender, single-parent families and delinquency: Exploring the moderating influence of race/ethnicity. Social Science Research, 35, 727-748.

* Forste, R., Bartkowski, J. P., \& Jackson, R. A. (2009). "Just be there for them": Perceptions of fathering among single, low-income men. Fathering, 7, 49-69.

* Gasser, R. D., \& Taylor, C. M. (1976). Role adjustment of single-parent families with dependent children. Family Coordinator, 25, 397-401.

Gates, G. J., Badgett, M. V. L., Macomber, J. E., \& Chambers, K. (2007). Adoption and foster care by gay and lesbian parents in the United States. Los Angeles, CA: Williams Institute; Washington, DC: Urban Institute. Retrieved from http://williamsinstitute.law.ucla.edu/wpcontent/uploads/Gates-Badgett-Macomber-Chambers-Final-AdoptionReport-Mar-2007.pdf

Gersick, K. E. (1979). Fathers by choice: Divorced men who receive custody of their children. In G. Levinger \& O. C. Moles (Eds.), Separation and divorce (pp. 307-323). New York, NY: Basic Books.

* Gladding, S. T., \& Huber, C. H. (1984). The position of the single-parent father. Journal of Employment Counseling, 21, 13-18.

* Grall, T. S. (2011, December). Custodial mothers and fathers and their child support: 2009. Current Population Reports. Washington, DC: US Census Bureau.

Green, C. (2010). Single custodial fathers and mothers meeting the challenge: A comparative note. In R. L. Coles \& C. Green (Eds.), The myth of the missing Black father (pp. 100-124). New York, NY: Columbia University Press.

*Greif, G. L., \& DeMaris, A. (1990). Single fathers with custody. Families in Society: The Journal of Contemporary Human Services, 71, 259-266.

*Guttman, J., \& Lazar, A. (1998). Mother's or father's custody: Does it matter for social adjustment? Educational Psychology: An International Journal of Experimental Educational Psychology, 18, 225-234. 
NOT THE PUBLISHED VERSION; this is the author's final, peer-reviewed manuscript. The published version may be

accessed by following the link in the citation at the bottom of the page.

*Hall, L. D., Walker, A. J., \& Acock, A. C. (1995). Gender and family work in one-parent households. J ournal of Marriage and the Family, 57, 685692.

* Hamer, J., \& Marchioro, K. (2002). Becoming custodial dads: Exploring parenting among low-income and working class African American fathers. Journal of Marriage and Family, 64, 116-129.

Hanson, S. M. H. (1981). Single custodial fathers and the parent-child relationship. Nursing Research, 30, 202-204.

* Hawkins, D. N., Amato, P. R., \& King, V. (2006). Parent-adolescent involvement: The relative influence of parent gender and residence. J ournal of Marriage and Family, 68, 125-136.

* Heath, D. T., \& Orthner, D. K. (1999). Stress and adaptation among male and female single parents. J ournal of Family Issues, 20, 557-587.

*Hill, L. C., \& Hilton, J. M. (1999). Changes in roles following divorce: Comparison of factors contributing to depression in custodial single mothers and custodial single fathers. J ournal of Divorce $\&$ Remarriage, 31, 91-114.

*Hilton, J., \& Devall, E. (1998). Comparison of parenting and children's behavior in single-mother, single-father, and intact families. Journal of Divorce \& Remarriage, 29, 23-54.

*Hilton, J. M., \& Koperafrye, K. (2007). Differences in resources provided by grandparents in single and married parent families. Journal of Divorce \& Remarriage, 47, 33-54.

* Hoffman, J. P., \& Johnson, R. A. (1998). A national portrait of family structure and adolescent drug use. Journal of Marriage and the Family, 41, 392- 407.

*Hook, J. L., \& Chalasani, S. (2008). Gendered expectations? Reconsidering single fathers' child-care time. Journal of Marriage and Family, 70, 978-990.

Jones, J., \& Mosher, W. D. (2013). Fathers' involvement with their children: United States, 2006-2010. National Health Statistics Reports. Washington, DC: US Department of Health and Human Services.

Koch, W. (2007, June 15). Number of single men adopting foster kids doubles. USA Today. Retrieved from http://usatoday30.usatoday.com/news/nation/2007-06-14foster n.htm

Kreider, R. M., \& Ellis, R. (2011, June). Living arrangements of children: 2009. Current Population Reports. Washington, DC: US Census Bureau.

Lareau, A. (1989). Home advantage: Social class and parental intervention in elementary education. New York, NY: Falmer Press. not grant permission for this article to be further copied/distributed or hosted elsewhere without the express permission from John Wiley \& Sons, Inc. 
NOT THE PUBLISHED VERSION; this is the author's final, peer-reviewed manuscript. The published version may be

accessed by following the link in the citation at the bottom of the page.

*Lee, S. M., \& Kushner, J. (2008). Single-parent families: The role of parent's and child's gender on academic achievement. Gender and Education, 20, 607-621.

Lin, T.-F., \& Chen, J. (2006). Custodial fathers-Do they work more or fewer hours? J ournal of Family Economic Issues, 27, 513-522.

* Livingston, G. (2013). The rise of single fathers. Pew Research Social \& Demographic Trends. Retrieved from http://www. pewsocialtrends.org/2013/07/02/the-rise-of-singlefathers/

Maccoby, E. E., \& Mnookin, R. H. (1992). Dividing the child: Social and legal dilemmas of custody. Cambridge, MA: Harvard University Press.

* McLanahan, S., \& Booth, K. (1989). Single mothers and their children: problems, reproduction, and politics. Journal of Marriage and the Family, 51, 557-580.

McLanahan, S., \& Bumpass, L. (1988). Intergenerational consequences of family disruption. American J ournal of Sociology, 93, 130-152.

McLanahan, S., \& Sandefur, G. (1994). Growing up with a single parent: What hurts, what helps. Cambridge, MA: Harvard University Press.

McNulty, T. L., \& Bellair, P. E. (2003). Explaining racial and ethnic differences in adolescent violence: Structural disadvantage, family well-being, and social capital. Justice Quarterly, 20, 1-31.

* Mendes, H. A. (1976a). Single fatherhood. Social Work, 21, 308-312.

* Mendes, H. A. (1976b). Single fathers. Family Coordinator, 25, 439-444.

* Meyer, D. R., \& Garasky, S. (1993). Custodial fathers: Myths, realities, and child support policy. Journal of Marriage and the Family, 55, 73-89.

Mulkey, L. M., Crain, R. L., \& Harrington, A. J. C. (1992). One-parent households and achievement. Sociology of Education, 65, 48-65.

Nord, C. W., Brimhall, D., \& West, J. (1997). Fathers' involvement in their children's schools. National Center for Education Statistics No. NCES 98-091. Washington, DC: US Department of Education.

* Nord, C. W., \& West, J . (2001). Fathers' and mothers' involvement in their children's schools by family type and resident status (National Household Education Survey: Statistical Analysis Report). Washington, DC: National Center for Education Statistics.

* Norton, A. J., \& Glick, P. C. (1986). One-parent families: A social and economic profile. Family Relations, 35, 9-17.

* Orthner, D. K., Brown, T., \& Ferguson, D. (1976). Single parent fatherhood: An emerging family life style. Family Coordinator, 25, 429-437.

* Orthner, D. K., \& Lewis, K. (1979). Evidence of single-father competence in child-rearing. Family Law Quarterly, 13, 27-47.

Pearson, J., Munson, P., \& Thoennes, N. (1982). Legal change and child custody awards. Journal of Family Issues, 3, 5-24.

Journal of Family Theory \& Review, Vol 7, No. 2 (June 2015): pg. 144-166. DOI. This article is (C John Wiley \& Sons, Inc. and permission has been granted for this version to appear in e-Publications@Marquette. John Wiley \& Sons, Inc does not grant permission for this article to be further copied/distributed or hosted elsewhere without the express permission from John Wiley \& Sons, Inc. 
* Powell, B., \& Downey, D. B. (1997). Living in single-parent households: An investigation of the same-sex hypothesis. American Sociological Review, 62, 521-539.

Rankin, J. H., \& Wells, L. E. (1994). Social control, broken homes, and delinquency. In G. Barak (Ed.), Varieties of criminology: Readings from a dynamic discipline (pp. 97-116). Westport, CT: Praeger.

* Risman, B. J. (1986). Can men "mother"? Life as a single father. Family Relations, 35, 95- 102.

* Risman, B. J. (1987). Intimate relationships from a microstructural perspective: Men who mother. Gender \& Society, 1, 6- 14.

* Risman, B. J., \& Park, K. (1988). Just the two of us: Parent-child relationships in single-parent homes. Journal of Marriage and Family, $5,1049-1062$.

* Rosenthal, K. M., \& Keshet, H. F. (1981). Fathers without partners: A study of fathers and family after marital separation. Totowa, NJ : Rowman \& Littlefield.

* Roy, K. (1999). Low-income single fathers in an African American community and the requirements of welfare reform. Journal of Family Issues, 20, 432-457.

*Santrock, J. W., \& Warshak, R. A. (1979). Father custody and social development in boys and girls. Journal of Social Issues, 35, 112-125.

*Smith, R. M., \& Smith, C. W. (1981). Child-rearing and single-parent families. Family Relations, 30, 411-417.

Taylor, P., Parker, K., Morin, R., Cohn, D., \& Wang, W. (2013). The new American father. Washington, DC: Pew Research Center. Retrieved from http://www.pewsocialtrends.org/files/2013/06/FINAL_Fathers report.p $\underline{\mathrm{df}}$

Thompson, R. A. (1983). The father's case in child custody disputes. The contributions of psychological research. In. M. E. Lamb \& A. Sagi (Eds.), Fatherhood and family policy (pp. 53-100). Hillsdale, NJ: Erlbaum.

*Thomson, E., McLanahan, S. S., \& Curtin, R. B. (1992). Family structure, gender, and parental socialization. Journal of Marriage and the Family, 54, 368-378.

US Census Bureau. (2011). Table 2. Historical living arrangements of children: 1880 to 2009 . Retrieved from http://www.census.gov/hhes/socdemo/children/data/sipp/living2009/t ables.html

US Census Bureau. (2013a). Table C3. Living arrangements of children under 18 years and marital status of parents, by age, sex, race, and Hispanic origin, and selected characteristics of the child for all children: $2013 a$. not grant permission for this article to be further copied/distributed or hosted elsewhere without the express permission from John Wiley \& Sons, Inc. 
NOT THE PUBLISHED VERSION; this is the author's final, peer-reviewed manuscript. The published version may be accessed by following the link in the citation at the bottom of the page.

Retrieved from www.census.gov/population/socdemo/hhfam/cps2006/tabC3

US Census Bureau. (2013). Table HH-1. Households, by type: 1940 to present. Current Population Survey. March and annual economic and social supplements, 2013 and earlier. Retrieved from http://www.census.gov/hhes/families/data/households.html

US Census Bureau. (2013b). Table FM-2. All parent/child situations, by type, race, and Hispanic origin of householder or reference person: 1970 to present. Current Population Survey. March and annual economic and social supplements, 2013 and earlier. Retrieved from http://www. census.gov/hhes/families/data/families.html

Warshak, R. A., \& Santrock, J. W. (1983). The impact of divorce in fathercustody and mother-custody homes: The child's perspective. In L. A. Kurdek (Ed.), Children and divorce: New directions for child development (pp. 29-45). San Francisco, CA: Jossey-Bass.

Weiss, R. S. (1979). Growing up a little faster: The experience of growing up in a single-parent household. Journal of Social Issues, 35, 97-111.

Zhan, M., \& Pandey, S. (2004). Post-secondary education and economic wellbeing of single mothers and single fathers. Journal of Marriage and Family, 66, 661-673. 